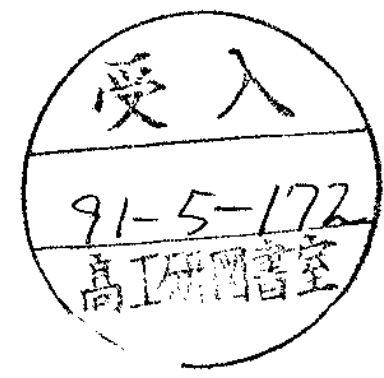

CERN-TH.6020/91

SU-ITP-888

KUL-TF-91/5

\title{
Superparticle actions and gauge fixings
}

\author{
E. Bergshoeff ${ }^{1}$ \\ Theory Division, CERN \\ CH-1211 Geneva 23 \\ R. Kallosh ${ }^{2}$ \\ Physics Department, Stanford University \\ Varian Bldg., Stanford CA 94305, USA \\ and A. Van Proeyen ${ }^{3}$ \\ Instituut voor Theoretische Fysica \\ Universiteit Leuven, B-3001 Leuven
}

\begin{abstract}
Recently, a covariant quantisation for the superparticle has been performed. Here we show the complete gauge-fixing procedure to obtain the correct BRST operator from the action proposed by one of us. This leads to a simpler form of this operator, revealing its structure. We also re-examine several previous attempts to perform a gauge fixing procedure of the Brink-Schwarz (BS) superparticle action. These all lead to a gauge-fixed action and a BRST operator which does not produce the correct physical spectrum. We clarify this issue by using the Batalin-Vilkovisky (BV) antibracket cohomology. The same gauge fixed action with a different BRST operator is the basis for the quantisation which gives the right number of physical states. From this BRST operator a classical action is reconstructed with an infinite number of classical fields and 2 infinite sets of symmetries which generalise the kappa symmetry of the BS action (the doubly infinite symmetric superparticle or DISP). By performing canonical transformations we bring the DISP action in a form which is close to the $\mathrm{BS}$ action. We comment about the differences between the two actions.

${ }^{1}$ Bitnet BERGSHOE at CERNVM

${ }^{2}$ On leave of absence from the Lebedev Physical Institute, Moscow, 117924 , USSR ; Bitnet KALLOSH at SLACVM

${ }^{3}$ Onderzoeksleider, N.F.W.O. Belgium; Bitnet FGBDA19 at BLEKUL11
\end{abstract}

CERN-TH.6020/91

SU-ITP-888

KUL-TF-91/5

March, 1991 


\section{Introduction}

Gauge theories play a crucial role in the description of the fundamental forces in physics. They all have in common the presence of unphysical degrees of freedom, physical quantities being by definition invariant under the local gauge symmetries of the theory. In the quantisation of a gauge theory these unphysical degrees of freedom are eliminated. In general this gauge fixing may spoil other global symmetries of the theory, like relativistic covariance. Covariant quantisation aims at leaving these global symmetries manifest.

The importance of covariant quantisation is that the global symmetries can be used to simplify the calculations in the quantum theory. For instance, it is expected that a covariant quantisation of the Green-Schwarz superstring model [1] will be helpful to prove the finiteness of the theory.

An important step towards covariant quantisation of gauge theories was taken by Faddeev and Popov [2]. They showed that for Yang-Mills gauge theories the gauge invariant action can be replaced by an "effective" action in the path integral which does not possess gauge invariances. Instead this "effective" action contains extra fields, the ghost fields. This method is not strictly applicable to an arbitrary gauge theory. Various people have generalised the Faddeev-Popov quantisation to more general gauge theories, and the Batalin-Vilkovisky (BV) formulation [3] encompasses all previous developments.

A covariant quantisation procedure for an arbitrary gauge theory has not yet been developed. During the last twenty years more and more examples have been found of gauge theories which cannot be quantised with the BV method, for instance superparticle, superstring and string field theories. A common feature of these theories is that quantisation seems to require the introduction of an infinite number of ghost fields. Gauge theories of this type are called infinite-reducible. The BV method in its present form can only be applied to gauge theories in which quantisation requires a finite number of ghost fields (the finite-reducible theories). The covariant quantisation of infinite-reducible gauge theories is an important open problem.

The simplest example of an infinite-reducible gauge theory is the BrinkSchwarz (BS) superparticle model [4], which is a supersymmetric extension of the standard relativistic particle. The infinite-reducibility of the model is due to a special symmetry, the so-called Siegel symmetry [5]. The BS superparticle has many properties in common with the superstring. It is therefore often used as a toy model to test new methods.

In the last two years, initiated by the work of W. Siegel [6], much research has been done on the covariant quantisation of the BS superparticle, but a truly covariant quantisation of the model has not been obtained. Starting from the classical BS superparticle action, a straightforward application of the $\mathrm{BV}$ formalism does not reproduce the correct physical spectrum, which is known from other, non-covariant, quantisation methods. Several modified actions have been proposed with the aim of circumventing this problem [7, 
$8,9,10,11,12]$.

In a recent work one of us [11] proposed a modified superparticle model (which has the same physical spectrum as the BS superparticle model) which can be covariantly quantised. This proposal is based on a modified BRST operator which gives the correct spectrum [10]. The modified superparticle action has two infinite sets of symmetries which generalise the Siegel symmetry of the BS action. The model is therefore called the doubly infinite symmetric superparticle or DISP.

It is the purpose of this paper to investigate the relationship between the BS and DISP superparticle models. We expect that a better insight into this relationship will be helpful in solving the problem of the covariant quantisation of the GS superstring model. We also hope that our investigations on the covariant quantisation of superparticle and superstring theories will teach us something about the quantisation of other infinite-reducible gauge theories as well and will lead to new insights in quantum field theory in general.

Although this paper mainly deals with superparticles we would like to put things in a more general setting and use this introduction to make some general remarks about the quantisation of gauge theories within the framework of the BV quantisation procedure. We will only discuss the Lagrangian approach to quantisation. The following discussion is inspired from [13, 14]. We refer to the latter for more details on the proofs and a lot of references to the original literature. We will illustrate the notions introduced below with the example of the bosonic particle.

In the Lagrangian approach a gauge theory is defined by the specification of an action $S_{0}(\phi)$ which is a functional of the classical fields $\phi^{i}$. The stationary surface is given by the set of all field configurations that satisfy the field equations. If the stationary surface contains only one point the theory is said to have no gauge degrees of freedom. Otherwise the theory contains gauge degrees of freedom. Given the action, the physical observables are defined as the solution to the field equations modulo gauge transformations. We only consider "regular" theories, which basically means that the standard loop expansion adopted in quantum field theory can be applied.

In a gauge theory there are relations between the stationary constraints or field equations. According to the nature of these relations gauge theories can be divided into three classes: irreducible, finite-reducible or infinitereducible. A gauge system is called irreducible if the functions relating the field equations form an independent set of functions. The system is called reducible if there are relations between these functions. These relations are given by another set of functions which themselves can form a dependent set, etc. If this process stops after a finite number of steps the system is called finite-reducible, otherwise it is called infinite-reducible.

The main problem in quantisation of a gauge theory is that the kinetic terms are not invertible and therefore the propagator cannot be defined. 
The aim of the quantisation procedure is to determine a new, gauge-fixed action, and to determine a BRST operator which leads to the same physical spectrum as the classical action.

The BV quantisation procedure provides a set of rules for how to achieve this. It consists of the following three essential steps.

1. Construct an "extended action". To obtain this we first extend the set of classical fields $\left\{\phi^{i}\right\}$ with several new fields (e.g. ghosts) to some set $\left\{\Phi^{A}\right\}$. Moreover for every field $\Phi^{A}$ we introduce an "antifield" $\Phi_{A}^{*}$ (of opposite statistics) and ghost number equal to

$$
g h\left(\Phi_{A}^{*}\right)=-g h\left(\Phi^{A}\right)-1
$$

(the classical fields have ghost number zero). The extended action is a function of all of these : $S\left(\Phi^{A}, \Phi_{A}^{*}\right)$ with ghost number zero. It should satisfy the following requirements

- $S_{c l}(\phi)=S(\Phi, 0)$ : the classical action is recovered when all antifields are set to zero.

- The extended action should satisfy a so-called master equation.

- $\mathcal{R}_{\alpha \beta} \equiv \vec{\partial}_{\alpha} \overleftarrow{\partial}_{\beta} S$ is a matrix of rank $N$ on the stationary surface, where $\alpha$ is a general notation for the fields and antifields $\left\{z^{\alpha}\right\}=$ $\left\{\Phi^{A}, \Phi_{A}^{*}\right\}$ and $N$ is the number of fields $\Phi^{A}$.

This will assure that the set of physical variables is represented by an antibracket $(\mathrm{AB})$ cohomology at ghost number zero.

$$
\text { phys. variables } \Rightarrow \mathrm{AB} \text { coho at ghost nr. } 0
$$

This means the following. One has defined an antibracket operation between functions. The antibracket cohomology is the cohomology of the operation which consists of taking an antibracket with the extended action $S$. (For precise definitions of these concepts see appendix A).

2. Add or subtract trivial sectors and perform canonical transformations. Trivial sectors are a set of fields with corresponding antifields for which terms are added or subtracted from the extended action which are separately solutions of the master equation and which carry no antibracket cohomology. Canonical transformations are invertible transformations in the set of fields and antifields which preserve canonical antibracket relations. This step should be performed to obtain a

3. gauge fixed action. This is an action $S\left(\Phi^{A}\right)$ obtained from the extended action at that point by putting all antifields equal to zero and which has $\operatorname{rank}\left(\mathcal{R}_{A B}\right)=N$, where $N$ is the number of fields which we have at this point. So $N$ non-trivial directions of the matrix $\mathcal{R}_{\alpha \beta}$ 
introduced in step 1 have been defined now as field directions. Then it can be proved that

$$
\mathrm{AB} \text { coho } \Rightarrow \text { BRST coho. }
$$

The antibracket cohomology is now the BRST cohomology for some operator

$$
\Omega \Phi^{A}=\left.\frac{\vec{\partial} S}{\partial \Phi_{A}^{*}}\right|_{\Phi^{*}=0} .
$$

The physical states are given by ghost number zero solutions of the field equations which are on-shell invariant under this BRST operator. Two such solutions are equivalent if they differ on-shell by a BRST exact term.

The second step of the procedure is not well determined. We did not say which trivial sectors to add or which canonical transformations to perform. Batalin and Vilkovisky did give such a prescription. However it is not always possible to find suitable functions which satisfy the conditions which they spelled out. On the other hand it is sometimes even not necessary to use their set of auxiliary fields.

To illustrate all this we now discuss the bosonic relativistic particle in $D$ dimensions as an example. The action defining the bosonic particle is given by:

$$
S_{0}(\phi)=P^{\mu} \dot{X}_{\mu}-\frac{1}{2} g P^{\mu} P_{\mu}
$$

The classical fields $\phi^{i}$ are the coordinates $X_{\mu}(\mu=1, \ldots, D)$, their conjugate momenta $P^{\mu}$ and the einbein $g$. The dot indicates a time derivative.

The above action is invariant under general coordinate transformations (with parameter $\xi$ ):

$$
\delta X^{\mu}=\xi P^{\mu}, \quad \delta g=\dot{\xi}
$$

The field equations corresponding to the action $S_{0}(\phi)$ are given by

$$
\dot{P^{\mu}}=0, \quad \dot{X}_{\mu}=g P_{\mu}, \quad P^{\mu} P_{\mu}=0
$$

Using the gauge transformations one can set $g=1$ and $X_{0}^{+}=P_{0}^{+}$where $X_{0}^{\mu}\left(P_{0}^{\mu}\right)$ indicate the constant (time-independent) modes of $X^{\mu}\left(P^{\mu}\right)$. In this gauge one can solve the field equations and one is left with the following set of physical variables:

$$
\left(X_{0}^{-}, P_{0}^{+}\right), \quad\left(X_{0}^{I}, P_{0}^{I}\right) \quad I=1,2, \ldots D-2
$$

The $D-1$ coordinates $\left\{X_{0}^{-}, X^{I}\right\}$ and their conjugate momenta $\left\{P_{0}^{+}, P_{0}^{I}\right\}$ describe a particle in $D$ dimensions.

The bosonic particle is an irreducible gauge theory: there is one relation between the first and third field equation given above and clearly the function 
describing this relationship is independent. The BV quantisation procedure tells us now that in order to go from the classical action to the extended action we need to introduce only a finite number of ghost variables. Following the exact rules we obtain the following extended action:

$$
S\left(\phi, \phi^{*}\right)=P^{\mu} \dot{X}_{\mu}-\frac{1}{2} g P^{\mu} P_{\mu}+X_{\mu}^{*} c P^{\mu}+g^{*} \dot{c} .
$$

Here $c$ is a new field, the ghost. The starred fields are the antifields. One can check that the 3 requirements from step 1 are satisfied. The transformation rules relevant for the antibracket cohomology $\mathcal{S} z=(z, S)$ (working from the right) are given by

$$
\begin{aligned}
\mathcal{S} P^{\mu} & =0 & & \mathcal{S} P_{\mu}^{*}=-\dot{X}_{\mu}+g P_{\mu}-X_{\mu}^{*} c \\
\mathcal{S} X^{\mu} & =c P^{\mu} & & S X_{\mu}^{*}=\dot{P}_{\mu} \\
\mathcal{S} g & =\dot{c} & & \mathcal{S} g^{*}=\frac{1}{2} P^{2}
\end{aligned}
$$

Note that $\mathcal{S}$ is nilpotent. i.e. $\mathcal{S}^{2}=0$. We have only given here those transformation properties which are relevant for the investigation of the antibracket cohomology at ghost number zero. Clearly, the solution to this cohomology is the same as the one given above: requiring that the fields are $\mathcal{S}$-invariant is equivalent to using the gauge invariance of the theory while requiring that the fields are not the $\mathcal{S}$ variation of something else is equivalent to imposing the field equations. The explicit form of the $\mathcal{S}$-invariant physical variables is given by:

$$
u^{\mu} \equiv X^{\mu}-\frac{P^{\mu}}{P^{+}} X^{+}
$$

where $u^{+}=0$. We then note that

$$
\mathcal{S}\left(P_{\mu}^{*}-\frac{P_{\mu}}{P^{+}} P^{+*}+\frac{X^{+}}{P^{+}} X_{\mu}^{*}-\frac{P_{\mu} X^{+}}{\left(P^{+}\right)^{2}} X^{+*}\right)=-\dot{u}_{\mu} .
$$

This shows that only the time independent part of the 9 variables $u_{\mu}$ is physical. The momenta are all $\mathcal{S}$-invariant. Furthermore $\dot{P}_{\mu}$ and $P^{2}$ are in the image of the $\mathcal{S}$ operation, and therefore we are left with 9 constant momenta variables. Note that in order to determine the antibracket cohomology it is not necessary that the extended action is gauge fixed: the antifield independent part of the extended action is invariant under general coordinate transformations.

Now we perform step 2. We first add a trivial sector to the extended action.

$$
S_{n m}=b^{*} d
$$

where $b$ is a fermionic 'antighost' and $d$ is a bosonic 'Lagrange multiplier'. Usually one chooses at this point a gauge fermion $\Psi(\Phi)$, and performs the substitutions

$$
\begin{aligned}
& \Phi^{A} \rightarrow \Phi^{A} \\
& \Phi_{A}^{*} \rightarrow \Phi_{A}^{*}+\partial_{A} \Psi(\Phi) .
\end{aligned}
$$


One can check that this is an example of a canonical transformation. In the present case we choose $\Psi(\Phi)=b(g-1)$. To bring the action in the desired form it is convenient to perform a further canonical transformation :

$$
\begin{array}{lll}
d & \rightarrow d+\frac{1}{2} P^{2}, & P_{\mu}^{*} \rightarrow P_{\mu}^{*}-d^{*} P_{\mu} \\
b & \rightarrow b-g^{*}, & g \rightarrow g-b^{*} .
\end{array}
$$

After performing this canonical transformations we end up with the following extended action:

$$
S=P^{\mu} \dot{X}_{\mu}-\frac{1}{2} P^{2}+b \dot{c}+X_{\mu}^{*} c P^{\mu}+\frac{1}{2} b^{*} P^{2}+(g-1) d .
$$

The last term is a trivial sector and can be omitted. We should stress that at this point the physical variables are still given by the antibracket cohomology of the extended action.

Now we are at step 3 of the BV procedure. The antifield independent part of the action is gauge fixed and the linear terms in antifields determine the following BRST transformations

$$
\Omega X^{\mu}=c P^{\mu}, \quad \Omega b=\frac{1}{2} P^{2}
$$

Note that $\Omega$, like $\mathcal{S}$ is nilpotent, i.e. $\Omega^{2}=0$.

In contradistinction to the antibracket cohomology we have to use the field equations in the analysis of the BRST cohomology. The field equations of the gauge-fixed action now determine only that $P^{\mu}$ is constant and determine everything except the zero modes of $X^{\mu}$. The constraint $P^{2}=0$ does not any more arise as a field equation, but as the BRST variation of the $b$ field. Finally the BRST variation of $X^{\mu}$ determines again that the physical variables are given by eq.(1.13).

It is often stated that the gauge fixed action is obtained in the BV formalism by imposing

$$
\Phi^{*}=\frac{\partial \Psi}{\partial \Phi}
$$

This is equivalent to first performing the canonical transformation eq.(1.16) and then putting $\Phi^{*}=0$. The latter formulation fits better in the philosophy that we want to keep antibrackets invariant at all stages, and thus we prefer to do canonical transformations.

In this paper we will apply the BV formalism, described above in the context of the bosonic particle, to the more complicated case of the BS and DISP superparticles. Both models are infinite-reducible and require the introduction of an infinite number of ghost fields. We should stress that the BV formalism in its present form is not meant to be applied to infinite-reducible systems. Nevertheless, the strategy so far has been to apply the existing 
BV formalism on infinite-reducible systems as well. The hope is that such applications may teach us something about how to modify, if necessary, the BV quantisation procedure.

We will start in section 2 by investigating the quantisation of the unmodified BS superparticle action. The classical action describes 8 physical fermionic degrees of freedom. It is the aim of the quantisation to have with the gauge fixed action a BRST operator whose cohomology leads to the same physical variables. To fix the Siegel symmetry we take a one-parameter family of gauge-fixings containing those which has been used in the previous years $[15,16,17,18]$. We find that in all cases there is a remaining gauge symmetry of the type discovered in $[19,16]$. We fix this symmetry by an extra gauge choice and obtain for all cases the same gauge fixed action and BRST transformations. This BRST operator does, however, not lead to the right physical states. We can show explicitly where its cohomology got modified. We will show this in the conclusion section. Nevertheless we want to present this calculation as it stands for the following reasons. First of all, the whole picture will give a clear illustration of the BV mechanism. But more important, the final (wrong) result leads us to consider the DISP particle action.

In fact, the field content of this gauge-fixed action looks the right one, which can be motivated by some counting arguments. The spinor fields in this action form two spinor representations of $O S p(10 \mid 4)[20,21,22]$. These have, according to some group theoretical counting rules, each a graded dimension equal to 4 . This would be the right number for producing the physical states of the classical action. However, the obtained BRST operator is trivial on the non-minimal fields. Only the ghost line and conjugate momenta occurred in a non-trivial way in this BRST operator. Such a line is a spinor representation of $O S p(10 \mid 2)$, and the same counting rules lead then to $8+8$ components, which is twice the number of physical variables of the classical action.

With this in mind, we have constructed in [10] a new BRST operator based on the same gauge-fixed action, and one of us has written a classical action which upon quantisation leads to this BRST operator. This is the DISP action mentioned before. In section 3 we will show how to obtain the DISP action from the BRST operator or the other way around. In fact there is a general way for first-order actions to interpolate between the classical action and the gauge-fixed one with its BRST operator. The fields in the DISP-BRST operator got in this way another meaning than they had in [10] (where they were considered as the ghost system of the BS action). This leads to a much simpler form.

In section 4 we will discuss the relationship between the BS and the DISP superparticle extended actions. We will perform canonical transformations to bring the DISP superparticle extended action in a form that resembles the BS superparticle extended action. We will comment about the differences between the two actions.

Finally in section 5 we will explain the discrepancy of the number of 
physical variables found in section 2 from the point of view of the antibracket cohomology. This section will also contain our conclusions.

There are three appendices. In appendix $\mathrm{A}$ we give our notations and conventions together with some more details about the BV formulation. In appendix $B$ we repeat the definitions of canonical transformations between fields and antifields and give some useful formulae for applying them. Finally, in Appendix $\mathrm{C}$ we illustrate some of the techniques of the $\mathrm{BV}$ formalism by means of the example of the antisymmetric tensor.

\section{The Brink-Schwarz superparticle}

In this section we will derive the gauge-fixed action and the BRST operator corresponding to the Brink-Schwarz (BS) superparticle action [4]. The final expressions for action and operator can be found in eq.(2.16) and eq.(2.17), respectively. These expressions will be derived by applying the Lagrangian BRST formalism of Batalin-Vilkovisky (BV) [3].

The gauge fixing procedure will start from the BS action [4], with its symmetries [5] and infinite set of zero modes of the latter [23]. Then we take a gauge choice which is an extension of the $\dot{\theta}=0$ gauge $[24,6]$. We consider a set of gauge choices, parametrised by a number $\alpha$, including the one in $[6,15](\alpha=1)$ and the one in [17] $(\alpha=0)$. We show explicitly at the end that all these gauge choices can be used to get the same final form after field redefinitions (one subtlety in the field redefinitions for $\alpha \neq 0$ will be discussed afterwards).

We will show how in a 2-step gauge procedure one can fix the symmetries of the superparticle action. After the first step the gauge fixed action still contains gauge symmetries $[19,16]$. These left over gauge invariances are of the "Stueckelberg" type, by which we mean that they effectively just eliminate a set of fields, in this case the "antighost branch". In a second step we will show how these symmetries are fixed.

The derivation of the result is given in subsection 2.1. There are a few subtleties which are skipped there. First of all, as mentioned above, after gauge fixing the kappa symmetries, we will find that the gauge-fixed action is still invariant under shift symmetries of the Stueckelberg type, which are then fixed in a second step. In subsection 2.1 these symmetries are fixed by an explicit gauge choice, which is proved in subsection 2.2 to be a regular procedure if one starts from a classical action with shift symmetries. However, we will argue in the conclusions (section 5) that this step is probably the cause of the truncation of the cohomology determining the physical states. Further, we will illustrate with the example of the antisymmetric tensor in appendix $\mathrm{C}$, that such a two-step gauge fixing sometimes leads to the correct cohomology, but can also lead to wrong results. It is not clear to us whether it is possible to choose a gauge fermion such that this second step gauge invariance can be avoided. 
Secondly, we will discuss in subsection 2.3 subtleties related to field redefinitions with an infinite set of variables. Such redefinitions were already questioned in $[19,16]$. In order to arrive at our final result for $\alpha=0$ we could avoid such dangerous field redefinitions. For $\alpha \neq 0$ we could also avoid them on the fields. However, if one considers also the antifields, the field redefinitions do not transform independent fields to new independent fields.

\subsection{The calculation with a general gauge}

Our starting point is the classical $d=10$ Brink-Schwarz (BS) superparticle action $[4]$

$$
S_{0}=P^{\mu} \dot{X}_{\mu}-\theta \not B \dot{\theta}-\frac{1}{2} g P^{\mu} P_{\mu}
$$

The classical variables are the coordinates $X_{\mu}$, their conjugate momenta $P^{\mu}$, the einbein $g$ and the fermionic variable $\theta$ which is a ten-dimensional Majorana-Weyl spinor. For simplicity we will in the following omit the tendimensional vector indices, e.g. $P^{\mu} P_{\mu}$ is indicated as $P^{2}$. The dot indicates a time derivative. Our further notation and conventions can be found in appendix A.

The action eq.(2.1) is invariant under general coordinate transformations (with parameter $\xi$ ) and fermionic so-called kappa transformations [5] (with parameter $\kappa)$ :

$$
\begin{aligned}
\delta X & =\xi P+\theta \gamma \not P \kappa \\
\delta \theta & =\not P \kappa \\
\delta g & =\dot{\xi}+4 \dot{\theta} \kappa
\end{aligned}
$$

The minimal solution to the master equation and the requirements of step 1 of the $\mathrm{BV}$ procedure mentioned in the introduction is given by [15]

$$
\begin{aligned}
S= & P \dot{X}-\theta \not p \dot{\theta}-\frac{1}{2} g P^{2} \\
& +X^{*}\left(c P+\theta \gamma \not P \theta_{1,0}\right)+g^{*}\left(\dot{c}+4 \dot{\theta} \theta_{1,0}\right) \\
& -2 c^{*} \theta_{1,0} \not \supset \theta_{1,0}+\sum_{p=0}^{\infty} \theta_{p, 0}^{*} \not P \theta_{p+1,0} \\
& +2 g^{*}\left[X^{*}\left(\theta \gamma \theta_{2,0}-\theta_{1,0} \gamma \theta_{1,0}\right)-4 c^{*} \theta_{1,0} \theta_{2,0}+\sum_{p=0}^{\infty} \theta_{p, 0}^{*} \theta_{p+2,0}\right]
\end{aligned}
$$

Here $c$ is the ghost corresponding to the general coordinate transformations, and $\left\{\theta_{p, 0}\right\}(p=1,2, \ldots)$ are the ghosts and ghosts for ghosts of the kappa symmetries and their zero modes. $\theta_{0,0} \equiv \theta$ is the classical field.

As written in the introduction for general gauge theories the physical variables are now given by the antibracket cohomology at ghost number zero. 
The variables invariant under $\mathcal{S} z=(z, S)$ are given by

$$
\begin{aligned}
& P^{\mu} \\
& U^{\mu} \equiv X^{\mu}-\frac{P^{\mu}}{P^{+}} X^{+}-\frac{1}{2} \frac{P^{\nu}}{P^{+}} \theta \gamma^{+\mu \nu} \theta-2 g^{*} \frac{1}{P^{+}} \theta \gamma^{+\mu} \theta_{1,0} \\
& s^{0,0} \equiv \not P \theta-2 g^{*} \theta_{1,0}
\end{aligned}
$$

where $\gamma^{\mu \nu \rho}=\gamma^{[\mu} \gamma^{\nu} \gamma^{\rho]}$ (with anti-symmetrisation of weight $1:[a b]=\frac{1}{2}(a b-$ $b a)$ ). Some of these are again in the image of the operation $\mathcal{S}$ :

$$
\begin{aligned}
\mathcal{S} g^{*} & =\frac{1}{2} P^{2} \\
\mathcal{S} X_{\mu}^{*} & =\dot{P}_{\mu} \\
\mathcal{S}\left(P_{\mu}^{*}\right. & -\frac{P_{\mu}}{P^{+}} P^{+*}+\frac{X^{+}}{P^{+}} X_{\mu}^{*}-\frac{P_{\mu} X^{+}}{\left(P^{+}\right)^{2}} X^{+*}-\frac{1}{2} \frac{P_{\nu}}{\left(P^{+}\right)^{2}} X^{+*} \theta \gamma^{+\mu \nu} \theta \\
& \left.-\frac{1}{2 P^{+}} \theta \gamma^{+\mu} \theta^{*}-\frac{1}{2 P^{+}} \theta_{1,0}^{*} \gamma^{+\mu} \theta_{1,0}-\frac{2}{\left(P^{+}\right)^{2}} g^{*} X^{+*} \theta \gamma^{+\mu} \theta_{1,0}\right)=-\dot{U}_{\mu} \\
\mathcal{S} g^{*} \theta & =-\frac{1}{2} \not P s^{0,0} \\
\mathcal{S}\left(\theta^{*}\right. & \left.+\theta X_{\mu}^{*} \gamma^{\mu}\right)=2 \dot{s}^{0,0} .
\end{aligned}
$$

We have thus the bosonic coordinates as in the introduction together with an additional eight constant fermionic degrees of freedom. This shows explicitly that the general theorem which states that the physical variables are represented by the antibracket cohomology is also satisfied in this case.

We go now to step 2 of the general BV procedure. We follow the scheme of Batalin and Vilkovisky [3] for introducing non-minimal sectors suitable for gauge fixings of reducible systems.

$$
S_{n m}=b^{*} d+\sum_{q=1}^{\infty} \sum_{p=q}^{\infty} \theta_{p, q}^{*} \lambda_{p, q}+\sum_{q=0}^{\infty} \sum_{p=q}^{\infty}\left(\bar{\theta}^{p, q}\right)^{*} \bar{\lambda}^{p, q}
$$

This contains for the general coordinate transformations the antighost $b$ and Lagrange multiplier $d$, and for the tower of spinor symmetries $\left\{\theta_{p, q}\right\}(p \geq$ $q ; q=1,2 \ldots)$, being extra ghosts, and $\left\{\bar{\theta}^{p, q}\right\}(p \geq q, q=0,1,2 \ldots)$, antighosts and extra antighosts. The non-minimal and extra ghosts have corresponding Lagrange multipliers $\left\{\lambda_{p, q}\right\}(p \geq q, q=1,2, \ldots)$. The antighosts have corresponding Lagrange multipliers $\left\{\bar{\lambda}^{p, q}\right\}(p \geq q, q=0,1,2, \ldots)$. The (anti-) commutativity properties of all fields are listed in appendix A. General facts about the BV formalism are repeated in appendix A.

In order to fix the general coordinate and kappa transformations we consider the following class of gauge fermions

$$
\Psi=b(g-1)+\sum_{q=0}^{\infty} \sum_{p=q}^{\infty}(-)^{p+q+1} \bar{\theta}^{p, q}\left(\dot{\theta}_{p, q}+\alpha \dot{\theta}_{p+1, q+1}\right) .
$$


For $\alpha=0$ this is the gauge of [17], while for $\alpha=1$ it is the gauge of [15]. The gauge fermion $\Psi$ can be viewed as the generating function of a canonical transformation. Performing this canonical transformation then leads to a new extended action. To obtain our final result we will have to perform a further set of canonical transformations which we will now describe.

First of all, since the first term in eq.(2.7) is an algebraic gauge condition on $g-1$, and as this field occurs only linearly in the extended action, we can apply lemma B.3 of the appendix. In our case this means the following. In the minimal part eq.(2.3) of the extended action we make the substitutions $g \rightarrow-b^{*}$ and $g^{*} \rightarrow b$. Furthermore in the non-minimal part $S_{n m}$ we omit the $b^{*} d$ term. The complete extended action then becomes

$$
\begin{aligned}
S= & P \dot{X}-\theta \not p \dot{\theta}-\frac{1}{2} P^{2}+b \dot{c}+4 b \dot{\theta} \theta_{1,0} \\
& +\sum_{p=0}^{\infty}\left(\theta_{p+1,0} \not P-2 \theta_{p+2,0} b\right) \dot{\bar{\theta}}^{p, 0} \\
& +\sum_{q=0}^{\infty} \sum_{p=q}^{\infty} \bar{\lambda}^{p, q}\left(\dot{\theta}_{p, q}+\alpha \dot{\theta}_{p+1, q+1}\right)+\sum_{q=1}^{\infty} \sum_{p=q}^{\infty}\left(\lambda_{p, q}+\alpha \lambda_{p+1, q+1}\right) \dot{\bar{\theta}}^{p, q} \\
& +X^{*}\left[c P+\theta \gamma \not p \theta_{1,0}-2\left(\theta \gamma \theta_{2,0}-\theta_{1,0} \gamma \theta_{1,0}\right) b\right] \\
& -2 c^{*} \theta_{1,0}\left(\not P \theta_{1,0}-4 \theta_{2,0} b\right) \\
& +\sum_{p=0}^{\infty} \theta_{p, 0}^{*}\left(\not P \theta_{p+1,0}-2 \theta_{p+2,0} b\right) \\
& +\frac{1}{2} b^{*} P^{2}+\sum_{q=1}^{\infty} \sum_{p=q}^{\infty} \theta_{p, q}^{*} \lambda_{p, q}+\sum_{q=0}^{\infty} \sum_{p=q}^{\infty}\left(\bar{\theta}^{p, q}\right)^{*} \bar{\lambda}^{p, q}
\end{aligned}
$$

Secondly, we observe that for all $\alpha$ the extended action is invariant under Stueckelberg symmetries. The reason is the following. In the classical action (the first three lines) $\bar{\theta}^{p, 0}$ occurs only in the second line and in the combination with the other $\vec{\theta}$ in the last term of the third line. If we add a total derivative to the second line, the dot acts there on $\theta, P$ or $b$, which gives in each case a field equation. For the $\dot{\theta}$ this is due to the relation

$$
\dot{\theta}_{p, 0}=\sum_{r=0}^{\infty} \frac{\delta_{\ell} S_{0}}{\delta \bar{\lambda}^{p+r, r}}(-\alpha)^{r}
$$

Therefore a shift of $\bar{\theta}^{p, 0}$ with $\bar{\epsilon}^{p}$ can be cancelled by a variation of other fields. To obtain invariance of the extended action we now choose the variations of the $\bar{\theta}^{p, q}$ for $q \geq 1$ such that the last term of the third line is by itself invariant. To be precise, we obtain the following Stueckelberg transformations :

$$
\begin{aligned}
\delta_{S T} X & =-\sum_{p=0}^{\infty} \theta_{p+1,0} \gamma \bar{\epsilon}^{p} \\
\delta_{S T} c & =2 \sum_{p=0}^{\infty} \theta_{p+2,0} \bar{\epsilon}^{p}(-)^{p+1}
\end{aligned}
$$




$$
\begin{aligned}
& \delta_{S T} \bar{\theta}^{p, q}=(-\alpha)^{q} \bar{\epsilon}^{p-q} \\
& \delta_{S T} \bar{\lambda}^{p, q}=(-\alpha)^{q}(-)^{p+q}\left[-\not P \bar{\epsilon}^{p-q-1}+2 b \bar{\epsilon}^{p-q-2}\right]
\end{aligned}
$$

This allows us to make the gauge choice $\vec{\theta}^{p, 0}=0$. Such gauge fixings of Stueckelberg symmetries are dealt with in subsection 2.2 where we will derive a general theorem dealing with such situations. In our specific example applying the theorem amounts to delete in eq.(2.8) all terms with $\bar{\theta}^{p, 0}$ and its antifield and adding terms of the form $\phi^{*} \delta_{S T}\left(\bar{\epsilon}^{p} \rightarrow \bar{\lambda}^{p, 0}\right) \phi$. The latter are related to the compensating transformations which are needed in order to stay in the gauge $\bar{\theta}^{p, 0}=0$. As a result we get

$$
\begin{aligned}
S= & P \dot{X}-\theta \not p \dot{\theta}-\frac{1}{2} P^{2}+b \dot{c}+4 b \dot{\theta} \theta_{1,0} \\
& +\sum_{q=0}^{\infty} \sum_{p=q}^{\infty} \bar{\lambda}^{p, q}\left(\dot{\theta}_{p, q}+\alpha \dot{\theta}_{p+1, q+1}\right)+\sum_{q=1}^{\infty} \sum_{p=q}^{\infty}\left(\lambda_{p, q}+\alpha \lambda_{p+1, q+1}\right) \dot{\bar{\theta}}^{p, q} \\
& +X^{*}\left[c P+\theta \gamma \not P \theta_{1,0}-2\left(\theta \gamma \theta_{2,0}-\theta_{1,0} \gamma \theta_{1,0}\right) b+\sum_{p=0}^{\infty} \bar{\lambda}^{p, 0} \gamma \theta_{p+1,0}\right] \\
& -2 c^{*}\left[\theta_{1,0}\left(\not P \theta_{1,0}-4 \theta_{2,0} b\right)+\sum_{p=0}^{\infty} \bar{\lambda}^{p, 0} \theta_{p+2,0}\right]+\frac{1}{2} b^{*} P^{2} \\
& +\sum_{p=0}^{\infty} \theta_{p, 0}^{*}\left(\not P \theta_{p+1,0}-2 \theta_{p+2,0} b\right)-\sum_{q=1}^{\infty} \sum_{p=q}^{\infty}\left(\bar{\theta}^{p, q}\right)^{*}(-\alpha)^{q} \bar{\lambda}^{p-q, 0} \\
& +\sum_{q=0}^{\infty} \sum_{p=q+1}^{\infty}\left(\bar{\lambda}^{p, q}\right)^{*}(-\alpha)^{q}\left[(-)^{p+q} \not \bar{\lambda}^{p-q-1,0}+2 \bar{\lambda}^{p-q-2,0} b\right] \\
& +\sum_{q=1}^{\infty} \sum_{p=q}^{\infty}\left[\theta_{p, q}^{*} \lambda_{p, q}+\left(\bar{\theta}^{p, q}\right)^{*} \bar{\lambda}^{p, q}\right]
\end{aligned}
$$

with as usual $\bar{\lambda}^{p, q}=0$ for $p<q$.

Thirdly, we eliminate the two terms with $\dot{\theta}$ in the first line using eq.(2.9) and corresponding redefinitions. The $\theta \not P \dot{\theta}$ term can be eliminated using the canonical transformation

$$
f=-\sum_{p=0}^{\infty}\left(\tilde{\tilde{\lambda}}^{p, p}\right)^{*}(-\alpha)^{p} \not \not \theta
$$

This generates a lot of extra terms in the transformation laws (in antifields). To eliminate the $b \theta \dot{\theta}_{1,0}$ term we perform the canonical transformation

$$
f=\sum_{p=0}^{\infty}(-\alpha)^{p}\left[(4-\beta)\left(\tilde{\bar{\lambda}}^{p, p}\right)^{*} \theta_{1}+\beta\left(\tilde{\bar{\lambda}}^{p+1, p}\right)^{*} \theta\right] b+\beta \tilde{c}^{*} \theta \theta_{1}
$$

where $\beta$ is arbitrary. In [15] and [16] this redefinition was used with $\beta=0$. We will use $\beta=2$ as it was done for the case $\alpha=0$ in [17]. The advantage of the $\beta=2$ choice is that in that case the canonical transformation eq. (2.13) 
eliminates all the terms introduced by the previous canonical transformation, as well as a lot of other terms in eq.(2.11). We now get ${ }^{1}$

$$
\begin{aligned}
S= & P \dot{X}-\frac{1}{2} P^{2}+b \dot{c} \\
& +\sum_{q=0}^{\infty} \sum_{p=q}^{\infty} \bar{\lambda}^{p, q}\left(\dot{\theta}_{p, q}+\alpha \dot{\theta}_{p+1, q+1}\right)+\sum_{q=1}^{\infty} \sum_{p=q}^{\infty}\left(\lambda_{p, q}+\alpha \lambda_{p+1, q+1}\right) \dot{\theta}^{p, q} \\
& +X^{*}\left[c P+\sum_{p=0}^{\infty} \bar{\lambda}^{p, 0} \gamma \theta_{p+1,0}\right]-2 c^{*} \sum_{p=0}^{\infty} \bar{\lambda}^{p, 0} \theta_{p+2,0}+\frac{1}{2} b^{*} P^{2} \\
& +\sum_{p=0}^{\infty} \theta_{p, 0}^{*}\left(\not p \theta_{p+1,0}-2 \theta_{p+2,0} b\right)-\sum_{q=1}^{\infty} \sum_{p=q}^{\infty}\left(\bar{\theta}^{p, q}\right)^{*}(-\alpha)^{q} \bar{\lambda}^{p-q, 0} \\
& +\sum_{q=0}^{\infty} \sum_{p=q+1}^{\infty}\left(\bar{\lambda}^{p, q}\right)^{*}(-\alpha)^{q}\left[(-)^{p+q} \not p \bar{\lambda}^{p-q-1,0}+2 \bar{\lambda}^{p-q-2,0} b\right] \\
& +\sum_{q=1}^{\infty} \sum_{p=q}^{\infty}\left[\theta_{p, q}^{*} \lambda_{p, q}+\left(\bar{\theta}^{p, q}\right)^{*} \bar{\lambda}^{p, q}\right] .
\end{aligned}
$$

Finally, we now get rid of the $\alpha$-dependence using the transformation based on the generating function

$$
\begin{aligned}
f= & \alpha \sum_{q=1}^{\infty} \sum_{p=q}^{\infty}\left[\tilde{\lambda}_{p, q}^{*} \lambda_{p+1, q+1}+\tilde{\theta}_{p, q}^{*} \theta_{p+1, q+1}\right] \\
& -\sum_{q=1}^{\infty} \sum_{p=q+1}^{\infty}\left(\tilde{\lambda}^{p, q}\right)^{*}(-\alpha)^{q} \bar{\lambda}^{p-q, 0}
\end{aligned}
$$

In conclusion, we find the following $\alpha$-independent result

$$
\begin{aligned}
S= & P \dot{X}-\frac{1}{2} P^{2}+b \dot{c}+\sum_{p=0}^{\infty} \bar{\lambda}^{p, 0} \dot{\theta}_{p, 0} \\
& +X^{*}\left[c P+\sum_{p=0}^{\infty} \bar{\lambda}^{p, 0} \gamma \theta_{p+1,0}\right]-2 c^{*} \sum_{p=0}^{\infty} \bar{\lambda}^{p, 0} \theta_{p+2,0}+\frac{1}{2} b^{*} P^{2} \\
& +\sum_{p=0}^{\infty} \theta_{p, 0}^{*}\left(\not B \theta_{p+1,0}-2 \theta_{p+2,0} b\right)+\left(\bar{\lambda}^{p, 0}\right)^{*}\left[(-)^{p} \not \bar{\lambda}^{p-1,0}+2 \bar{\lambda}^{p-2,0} b\right] \\
& +\sum_{q=1}^{\infty} \sum_{p=q}^{\infty}\left[\bar{\lambda}^{p, q} \dot{\theta}_{p, q}+\lambda_{p, q} \dot{\bar{\theta}}^{p, q}+\theta_{p, q}^{*} \lambda_{p, q}+\left(\bar{\theta}^{p, q}\right)^{*} \bar{\lambda}^{p, q}\right] .
\end{aligned}
$$

The redefinition eq.(2.15), which only has to be made if $\alpha \neq 0$, is an "allowed redefinition" on the fields (i.e. it does not lead to constraints on the new fields), but the corresponding redefinition on antifields is troublesome. We will discuss this issue in subsection 2.3.

\footnotetext{
${ }^{1}$ It is understood that we omit the tildes after the canonical transformations are performed.
} 
The final result given in eq.(2.16) gives the following information. The terms independent of antifields both determine the Hamiltonian to be $\frac{1}{2} P^{2}$ and determine which are the coordinates and their conjugate momenta. From the terms linear in the antifields one then derives the BRST operator $\Omega$ (using eq.(A.6)) :

$$
\Omega_{0}=\frac{1}{2} c P^{2}+\sum_{p=0}^{\infty} \bar{\lambda}^{p, 0}\left(\not P \theta_{p+1,0}-2 \theta_{p+2,0} b\right)+\sum_{q=1}^{\infty} \sum_{p=q}^{\infty} \bar{\lambda}^{p, q} \lambda_{p, q} .
$$

The fact that there are no terms quadratic in antifields implies that this operator is nilpotent off-shell.

The gauge fixed action given in eq.(2.16) describes the correct number of $8+8$ (bosonic + fermionic) field degrees of freedom, like in the lightcone gauge. Following the arguments of [22] this counting of field degrees of freedom guarantees that the vacuum to vacuum amplitude in the covariant gauge is the same as in the light-cone gauge. Note that the non-minimal sector ( $q \geq 1$ fields), which contributes to the above counting of field degrees of freedom, splits off completely from the minimal sector and has no solutions to the equations $\Omega|\phi>=0 ;| \phi>\neq \Omega \mid \chi>$. This can be seen as the reason why the cohomology of this BRST operator does not give the right number of physical states. This result lead us in [10] to keep the same gauge-fixed action, but to introduce another BRST operator, where these non-minimal fields enter non-trivially, and which will be discussed in the next section.

\subsection{Stueckelberg with or without ghosts}

In this subsection we show how to fix shift symmetries of the Stueckelberg type. We first show a toy model with the simplest form of a shift symmetry. Then we explain how in the general case of a classical action with Stueckelberg symmetries the latter can be fixed with or without the introduction of corresponding ghosts (with the same final result).

By a Stueckelberg symmetry we understand that we can do local field redefinitions such that only one field transforms under that symmetry, under which it undergoes a shift. The simplest example where this happens is the following toy model. We have an action $S_{0}(A, B)=S_{0}(B)$. In other words we have introduced $A$ as a variable in the path integral, but it does not occur in the action. The physical content which we want to obtain is the theory in terms of only $B$. So we have to consider the states invariant under the 'Stueckelberg symmetry'

$$
\delta A=\epsilon
$$

There are two ways to handle this situation. The trivial method without ghosts consists thus in imposing a 'gauge choice' $A=0$. The second way is to include a fermionic ghost $c$ and to add to the extended action the term giving the gauge transformation

$$
S=S_{0}(B)+A^{*} c
$$


Now the last term is a trivial term in the BV terminology. In other words the integration in the path integral over $A$ is removed by deleting the fields $\left(A, A^{*}, c, c^{*}\right)$. Clearly this quartet [25] does not contribute to the antibracket cohomology as $\mathcal{S} A=c ; \mathcal{S} c^{*}=A^{*}$. We thus conclude that we can choose to either include the corresponding ghost system, or to do the gauge fixing by putting $A=0$ at the classical level. Such commutativity between algebraic gauge fixing and quantisation was already noticed in some other situation in [26].

In general the situation can be a bit more complicated in the sense that after fixing the Stueckelberg symmetry both the BRST transformations (i.e. the terms of the extended action linear in the antifields) as well as all the relevant non-closure functions (corresponding to the terms of the extended action higher order in the antifields) can change. We will now derive a theorem which gives what these changes are for a wide class of general extended actions. To be specific, we consider fields $\lambda^{p}$ which are shifted under (anti)commuting symmetry transformations. In the extended action we include these transformations using ghosts $c^{p}$. All other fields are denoted by $\phi$. We now prove the following theorem.

Theorem 2.1 Consider the following general possibility

$$
\begin{aligned}
S= & \left(c^{p}+A_{2}^{p}\left(\phi, \phi^{*}\right)\right) \lambda_{p}^{*}+c^{p} A_{1 p}\left(\phi, \phi^{*}\right) \\
& +U^{p}\left(c, c^{*}, \phi, \phi^{*}\right) c_{p}^{*}-\lambda^{p} T_{p}\left(c, c^{*}, \phi, \phi^{*}\right)+U_{0}\left(\phi, \phi^{*}\right)
\end{aligned}
$$

where, moreover

$$
\left.\frac{\partial T_{p}}{\partial c^{q}}\right|_{c^{*}=0}=0 .
$$

This extended action can be brought into the following simple form :

$$
S=U_{0}\left(\phi, \phi^{*}\right)-A_{2}^{p}\left(\phi, \phi^{*}\right) A_{1 p}\left(\phi, \phi^{*}\right)
$$

The proof relies on the lemmas of appendix B. We choose the algebraic gauge condition $\lambda^{p}=0$ such that we can start by using lemma B.3. This introduces the antighosts $b_{p}$, and we get

$$
\begin{aligned}
S= & \left(c^{p}+A_{2}^{p}\left(\phi, \phi^{*}\right)\right) b_{p}+c^{p} A_{1 p}\left(\phi, \phi^{*}\right) \\
& +U^{p}\left(c, c^{*}, \phi, \phi^{*}\right) c_{p}^{*}+b^{* p} T_{p}\left(c, c^{*}, \phi, \phi^{*}\right)+U_{0}\left(\phi, \phi^{*}\right) .
\end{aligned}
$$

We next observe that the ghost-antighost system is auxiliary and therefore we can eliminate it using lemma B.1. One can verify that the condition eq.(B.6) is satisfied if eq.(2.21) holds, and that after applying the lemma, eq. (2.22) is obtained.

Note that for the application of the lemmas B.3 and B.1 one does not have to know the explicit form of the functions $U^{p}$. One only has to check whether the extended action is of the general form given by the theorem and 
whether the condition eq.(2.21) is satisfied. It is known that if one imposes a gauge and eliminates gauge transformations, one has to add compensating transformations to remain in that gauge. The power of the above theorem is that it not only tells what these compensating transformations are but also automatically takes care of the corrections to the non-closure functions.

\section{$2.3 \quad$ Field redefinitions}

In the calculations of subsection 2.1 we have made many field redefinitions. But only one of them, eq. (2.15) (non-trivial only for $\alpha \neq 0$ ), is related to the problems mentioned in $[19,16]$. In this subsection we would like to discuss some of the subtleties which are involved in making such redefinitions. We want to distinguish between the following two types of infinite field redefinitions.

type 1 : We go from an infinite set of fields $\theta_{p}(p=0,1, \ldots)$ to another set $\tilde{\theta}_{p}(p=0,1, \ldots)$ by

$$
\tilde{\theta}_{p}=\theta_{p}+\theta_{p+1}
$$

The inverse of this is

$$
\theta_{p}=\sum_{r=0}^{\infty}(-)^{r} \tilde{\theta}_{p+r}
$$

Assuming that the $\theta_{p}$ are independent this redefinition leads to unconstrained variables. Specifically the equation

$$
\sum_{p=0}^{\infty} c_{p} \tilde{\theta}_{p}=0
$$

leads to the relations $c_{p}=c_{p-1}$ for $p \geq 1$ and $c_{0}=0$, and hence $c_{p}=0$ for all $p$. So there is no constraint.

type 2: We now consider the redefinitions

$$
\tilde{\theta}_{p}=\theta_{p}+\theta_{p-1}
$$

where $\theta_{-1}=0$. The inverse of this redefinition is

$$
\theta_{p}=\sum_{r=0}^{p}(-)^{r} \tilde{\theta}_{p-r}
$$

However, now the equation

$$
\sum_{p=0}^{\infty} c_{p} \tilde{\theta}_{p}=0
$$

with independent $\theta_{p}$ variables gives $c_{p}=c_{p+1}$ for $p \geq 0$, so we get the solution

$$
c_{0}=-c_{1}=c_{2}=\ldots \neq 0
$$


and thus the constraint

$$
\sum_{p=0}^{\infty} \tilde{\theta}_{p}(-)^{p}=0
$$

Therefore we want to avoid redefinitions of this second type as they do not lead to independent new variables. However, even for the first type a further subtlety occurs which we will discuss now.

\section{Canonically conjugate variables.}

Consider a set of variables $\theta_{p}$ together with their canonical conjugates $\pi^{p}$. These conjugates can be either antifields or conjugate momenta. There is thus a bracket structure with

$$
\left[\theta_{p}, \pi^{q}\right\}=\delta_{p}^{q}
$$

Suppose that we perform a redefinition of type 1 on the fields $\theta_{p}$, then the canonical conjugates of the new variables are

$$
\tilde{\pi}_{p}=\sum_{r=0}^{p}(-)^{r} \pi_{n-r}
$$

This is the redefinition eq.(2.28), i.e. the inverse of a redefinition of type 2 .

On the other hand, if we would perform a redefinition of type 2 on the fields $\theta_{p}$ we would get

$$
\tilde{\pi}_{p}=\sum_{r=0}^{\infty}(-)^{r} \pi_{p+r}
$$

which is the inverse of a redefinition of type 1.

We conclude that the canonical conjugates of type 1 redefinitions lead to the inverse of type 2 redefinitions and vice versa. These inverses do not immediately lead to constraints, but the fact that the inverse of these redefinitions gives constraint variables implies that one cannot treat the new and the old variables as independent.

The redefinition eq.(2.15) is for $\alpha \neq 0$ of the type 1 for the fields, and thus of the type 2 for the antifields (for $\alpha=0$ this redefinition is absent). Hence for $\alpha \neq 0$ we do find unconstrained variables but constrained antifields. The consequences of this fact are unclear to us.

We would like to close this subsection with the following two remarks. First of all, in order to avoid making redefinitions of the type 2 on fields it was crucial that in the diagonalisation of the sum

$$
\sum_{p=0}^{\infty} \lambda^{p}\left(\theta_{p}+\theta_{p-1}\right)
$$

we first rewrote this sum as

$$
\sum_{p=0}^{\infty}\left(\lambda^{p}+\lambda^{p+1}\right) \theta_{p}
$$


Then we performed a field redefinition of the first type to diagonalise the sum.

Secondly, in deriving the results of subsection 2.1 we not only performed field redefinitions but also manipulated infinite summations. Our infinite summations were not rigorously defined by the explicit use of a regulator. Therefore we cannot claim that the formal manipulations on the infinite sums of fields, which we performed above, are well-defined with strict mathematical rigor. In a more rigorous approach one should from the beginning define the meaning of the infinite sum of fields, e.g. by the limit of a finite sum to $p=N$ and then taking the limit $N \rightarrow \infty$. Clearly, it is getting time that an extension of the BV formalism in full rigor is developed which is able to deal with infinite reducible theories like superparticles and superstrings.

\section{The DISP action}

The problems mentioned in the previous section (the BRST operator did not give the right cohomology) lead us to construct [10] another BRST operator keeping the same gauge fixed action

$$
S_{0, g f}=P \dot{X}-\frac{1}{2} P^{2}+b \dot{c}+\sum_{q=0}^{\infty} \sum_{p=q}^{\infty}\left[\bar{\lambda}^{p, q} \dot{\theta}_{p, q}+\lambda_{p+1, q+1} \dot{\bar{\theta}}^{p+1, q+1}\right] .
$$

The total extended action is thus of the form (see appendix A for the definition of the Dirac brackets)

$$
S_{g f}=\frac{1}{2} \Phi^{I} C_{I J} \dot{\Phi}^{J}-H_{0}+\Phi_{I}^{*}\left[\Phi^{I}, \Omega\right] .
$$

The new BRST operator is given by

$$
\begin{aligned}
\Omega & =\frac{1}{2} c P^{2}+\sum_{q=0}^{\infty} \sum_{p=q}^{\infty} \bar{\lambda}^{p, q}\left(\not p \theta_{p+1, q}-2 \theta_{p+2, q} b\right) \\
& +\sum_{q=1}^{\infty} \sum_{p=q+1}^{\infty} \lambda_{p+1, q}\left(\not p \bar{\theta}^{p, q}-2 \bar{\theta}^{p-1, q} b+\left(\bar{\lambda}^{p+1, q}+\bar{\lambda}^{p, q-1}\right)(-)^{\frac{1}{2}(p-q+1)(p-q)}\right) \\
& +\sum_{p=1}^{\infty} \lambda_{p+1, p}\left(\not p \bar{\theta}^{p, p}+\left(\bar{\lambda}^{p+1, p}+\bar{\lambda}^{p, p-1}\right)-2 b\left(\theta_{p, p}-\theta_{p-1, p-1}\right)\right) \\
& +\sum_{p=0}^{\infty} \lambda_{p+1, p+1}\left(\bar{\lambda}^{p+1, p+1}-\not p \theta_{p+1, p+1}+2 \theta_{p+2, p+1} b+\bar{\lambda}^{p, p}+\not p \theta_{p, p}-2 \theta_{p+1, p} b\right) .
\end{aligned}
$$

In [11] one of us gave a classical action which upon quantisation reproduces the gauge-fixed action and BRST operator above. In this section we would like to clarify how precisely this relation is obtained. The classical superparticle action of [11] is given by :

$$
S_{0}=P \dot{X}-\frac{1}{2} g P^{2}+\sum_{p=0}^{\infty} \lambda^{p} \dot{\theta_{p}}
$$




$$
-\sum_{p=0}^{\infty} \lambda^{p} \not \not \zeta^{p}-\sum_{p=0}^{\infty}\left\{\lambda^{p+1}-\theta_{p+1} \not P+\lambda^{p}+\theta_{p} \not P\right\} \eta_{p}
$$

It was shown in [10] that the BRST operator eq.(3.3) has only 4 fermionic coordinates and 4 momenta as solution of its cohomology equations, and thus gives the correct physical states. The same methods as used there, can be applied to the analysis of the physical variables described by the classical action eq.(3.4). The symmetries of the action has been given in [11]. The bosonic part goes of course as in the introduction. For the fermionic part, we first choose the gauge $\zeta^{p}=\eta_{p}=0$ leaving only the zero modes of the corresponding transformations unfixed. Then the field equations of $\theta_{p}$ and $\lambda^{p}$ imply that they are both constants. The remaining field equations are

$$
K_{p} \equiv \not \not \lambda^{p}=0 ; \quad F^{p} \equiv \lambda^{p+1}-\not \not \theta_{p+1}+\lambda^{p}+\not p \theta_{p}=0
$$

and the constant remaining gauge transformations are given by

$$
\delta \lambda^{p}=\not P\left(\xi_{p}-\xi_{p-1}\right) ; \quad \delta \theta_{p}=\not p \kappa^{p}+\left(\xi_{p}+\xi_{p-1}\right) .
$$

We go in the frame where $P^{\mu}$ has only the component $P^{+}$. Then we split the spinors in, e.g.,

$$
\theta=\frac{1}{2}\left(\gamma^{+} \gamma^{-}+\gamma^{-} \gamma^{+}\right) \theta \equiv \gamma^{+} \theta^{-}+\gamma^{-} \theta^{+}
$$

The first field equation puts $\lambda^{-p}=0$ while the $\kappa^{p}$ transformations just perform a shift on $\theta_{p}^{+}$and can thus be used to eliminate these variables. We are thus left at this point with constant spinors $\lambda^{+p}$ and $\theta_{p}^{-}$. It is now convenient to combine them in

$$
q^{+p}=\lambda^{+p}-\gamma^{+} P^{+} \theta_{p}^{-} ; \quad \tilde{q}^{+p}=\lambda^{+p}+\gamma^{+} P^{+} \theta_{p}^{-}
$$

The remaining field equations leaves us with $\tilde{F}^{p}=q^{+p+1}-\tilde{q}^{+p}(p=0,1, \ldots)$ and $q^{+0}$. The remaining $\xi$ symmetries act only as shift symmetries on $\tilde{F}^{p}$ which can hence be discarded. The $q^{+0}$ are thus the eight fermionic physical variables.

The action eq.(3.4) is a first-order action of the general form

$$
S_{0}=\frac{1}{2} \phi^{i} C_{i j} \dot{\phi}^{j}-T_{a}(\phi) \psi^{a}-H_{0}(\phi)
$$

where the classical fields are $\left\{\phi^{i}\right\}=\left\{X^{\mu}, P_{\mu}, \theta_{p} \equiv \theta_{p, p}, \lambda^{p} \equiv \bar{\lambda}^{p, p}\right\}$ and the Lagrange multipliers $\psi^{a}=\left\{\zeta^{p}, \eta_{p}\right\}$. In this expression $C_{i j}$ is a constant (invertible) matrix and the $T_{a}$ are functions of the classical fields ("constraints") which form a first-class algebra (see below). This is different from the BS superparticle action. The latter involves both first- and second-class constraints which cannot be separated covariantly. 
The BS action has a $\kappa$ symmetry. The action eq.(3.4) has two infinite sets of symmetries. Therefore we will call it the "Doubly Infinite Symmetric superParticle" or DISP. In the BS action the infinite number of fields are related to the infinite reducibility of the symmetries. For the DISP there are already an infinite number of classical fields and of first-order ghosts related to the symmetries, which are moreover infinite reducible.

We will derive in general a relationship between first-order actions of the form eq.(3.9) and their corresponding extended action eq.(3.2) and BRST operator. We will not only show how to obtain the extended action and BRST operator starting from the classical action given in eq.(3.9) but moreover we will show how, given a BRST operator, the corresponding classical action can be reconstructed.

The organisation of this section is as follows. To establish the notations we will first consider the case that the action eq.(3.9) is irreducible. We next consider the 1-stage reducible case. We then immediately jump to the case where the action eq.(3.9) corresponds to an infinite reducible system. We finally apply the latter case to the DISP action eq.(3.4) and show that the BRST operator eq.(3.3) is indeed reproduced by this classical action. The general scheme for going from a Lagrangian formulation to the BRST operator or the other way around has been given first in [27] (for a review, see also [14]).

\subsection{First-order action Lagrangian quantisation for ir- reducible systems}

Following the BV formalism it is a relatively easy matter to establish that in the irreducible case the extended action corresponding to eq.(3.9) is given $\mathrm{by}^{2}$

$$
\begin{aligned}
S^{i r r}= & \frac{1}{2} \phi^{i} C_{i j} \dot{\phi}^{j}-T_{a} \psi^{a}-H_{0} \\
& +\phi_{i}^{*}\left[\phi^{i}, T_{a}\right] c^{a}+\psi_{a}^{*}\left(\dot{c}^{a}+f_{b c}^{a} \psi^{c} c^{b}\right)+\frac{1}{2} c_{a}^{*} f_{b c}^{a} c^{c} c^{b}(-)^{b}
\end{aligned}
$$

Here $c^{a}$ are the ghosts and $C^{i j}$ is the inverse matrix of $C_{i j}: C_{i j} C^{j k}=\delta_{i}^{k}$. We can thus define brackets as in appendix A. The $f_{b c}^{a}$ are the structure functions which are defined by $\left[T_{a}, T_{b}\right]=T_{c} f_{a b}^{c}$. We assume that these structure functions are constants and satisfy the Jacobi identity. We assume that $H_{0}$ has zero bracket with the $T_{a}$. The last line in eq.(3.10) is the nonminimal part.

For gauge fixing in the Lagrangian formalism one usually introduces a non-minimal sector and a gauge fermion

$$
S^{n m}=b^{* a} \lambda_{a} ; \quad \Psi=b_{a} \psi^{a}
$$

\footnotetext{
$b_{a}$

${ }^{2}$ When we write $(-)^{a}$ we denote by $a$ the statistics of $T_{a}$ or $\psi^{a}$, thus opposite to $c^{a}$ or
} 
where $b_{a}$ the anti-ghosts and $\lambda_{a}$ the Lagrange multipliers. Using our lemma B.3, this implies that one should replace in the extended action.

$$
\begin{aligned}
& \psi^{a} \rightarrow-b^{* a} \\
& \psi_{a}^{*} \rightarrow b_{a} .
\end{aligned}
$$

Another way of formulating this gauge-fixing procedure is the following. We do not introduce a non-minimal sector $\left(b_{a}, \lambda^{a}\right)$, but just perform the canonical transformation eq. (3.12).

The extended action then becomes

$$
\begin{aligned}
S_{g f}^{i r r}= & \frac{1}{2} \phi^{i} C_{i j} \dot{\phi}^{j}+T_{a} b^{* a}-H_{0} \\
& +\phi_{i}^{*}\left[\phi^{i}, T_{a}\right] c^{a}+b_{a}\left(\dot{c}^{a}-f_{b c}^{a} b^{* c} c^{b}\right)+\frac{1}{2} c_{a}^{*} f_{b c}^{a} c^{c} c^{b}(-)^{b} .
\end{aligned}
$$

It is of the form eq.(3.2) with the BRST operator $\Omega$ given by

$$
\Omega=T_{a} c^{a}+\frac{1}{2} b_{a} f_{b c}^{a} c^{c} c^{b}(-)^{b} .
$$

The last term in eq. (3.2) can also be written as $\left[\Omega, \Phi^{I}\right] \Phi_{I}^{*}$. The set $\{\Phi\}$ includes the extra conjugate pair $\left(c^{a}, b_{a}\right)$ which satisfy the bracket relations

$$
\left[c^{a}, b_{b}\right]=\delta_{b}^{a} .
$$

The $\Omega$ given in eq.(3.14) satisfies $[\Omega, \Omega]=0$ automatically since it is derived from an extended action which satisfies the master equation.

One can also go the other way round. From the BRST operator given in eq. (3.14) one can rederive the extended action given in eq.(3.10) as follows. We first use eq.(3.2). Next we undo the gauge-fixing by performing the substitutions

$$
\begin{aligned}
b_{a} & \rightarrow \psi_{a}^{*} \\
b^{* a} & \rightarrow-\psi^{a}
\end{aligned}
$$

which are just the inverse of the canonical transformation given in eq.(3.12). Making these substitutions in the BRST operator gives rise to a fermion

$$
\Omega^{\prime}=T_{a}(\phi) c^{a}+\frac{1}{2} \psi_{a}^{*} f_{b c}^{a} c^{c} c^{b}(-)^{b} .
$$

This gives then rise to the following extended action before gauge fixing :

$$
S^{i r r}=\frac{1}{2} \phi^{i} C_{i j} \dot{\phi}^{j}-H_{0}+\psi_{a}^{*} \dot{c}^{a}+\phi_{i}^{*}\left[\phi^{i}, \Omega^{\prime}\right]+c_{a}^{*} \frac{\partial_{l}}{\partial \psi_{a}^{*}} \Omega^{\prime}-\frac{\partial_{r}}{\partial c^{a}} \Omega^{\prime} \psi^{a} .
$$

which is of course nothing else than the extended action given in eq.(3.10) (without the non-minimal term).

We will now proceed with generalizing the above formulae to the case of a 1-stage reducible system. 


\subsection{First-order action Lagrangian quantisation for 1- stage reducible systems}

In a 1-stage reducible system the above formulae have to be modified due to the presence of zero modes. These zero modes are characterised by functions $Z^{a}{ }_{a_{1}}$ as follows:

$$
T_{a} Z_{a_{1}}^{a}=0 .
$$

We assume here that all zero modes are included. This has the implication ${ }^{3}$ :

$$
T_{a} X^{a}=0 \Rightarrow X^{a}=Z_{a_{1}}^{a} \epsilon^{a_{1}}+T_{b} E^{b a}
$$

for some $\epsilon^{a_{1}}$ and for certain functions $E^{a b}$ with which have the symmetry properties $E^{a b}=(-)^{a b+1} E^{b a}$.

To keep our formulae simple we will now make a few assumptions. All these assumptions are valid in case of the DISP action. First of all we will restrict ourselves to the case where $E^{a b}=0$. Secondly we assume that the following equation holds ${ }^{4}$ :

$$
f^{a}{ }_{c b} \psi^{b} Z_{a_{1}}^{c}-\left[T_{b} \psi^{b}, Z_{a_{1}}^{a}\right]=0
$$

for arbitrary $\psi$. In the general case one can prove (from taking the bracket of eq.(3.19) with $T_{b} \psi^{b}$ and using eq.(3.20)) that the 1.h.s. is proportional to a zero mode and a field equation $T_{a}$ defining "new" structure functions $f^{a_{1}} b_{b_{3}}$ and non-closure functions $E_{c a_{1}}^{a b}$. We assume that these functions are zero.

The fact that there are zero modes has two implications. First of all it implies that the classical action eq.(3.9) has additional symmetries. These new symmetries require the addition of new terms to the extended action involving the ghosts for these new symmetries which we denote by $\check{c}^{a_{1}}$. Secondly, due to the zero modes, we must also introduce new terms involving the ghosts for ghosts $c^{a_{1}}$. Below, we summarise the statistics of these ghosts

\begin{tabular}{lc|cccc} 
& ghost number & field & statistics & field & statistics \\
\hline ghosts & 1 & $c^{a}$ & $(-)^{a+1}$ & $\check{c}^{a_{1}}$ & $(-)^{a_{1}+1}$ \\
ghosts for ghosts & 2 & $c^{a_{1}}$ & $(-)^{a_{1}}$ & &
\end{tabular}

We thus end up with the following extended action corresponding to a 1-stage reducible system:

$$
S^{1 \text { red }}=S^{i r r}+\psi_{a}^{*} Z^{a}{ }_{a_{1}} \check{c}^{a_{1}}-c_{a}^{*} Z^{a}{ }_{a_{1}} c^{a_{1}}+\check{c}_{a_{1}}^{*} \dot{c}^{a_{1}}+\psi_{a}^{*} \phi_{i}^{*}\left[\phi^{i}, Z^{a}{ }_{a_{1}}\right] c^{a_{1}}(-)^{a}
$$

(Here $S^{i r r}$ is given by eq.(3.10)). Of course we have also assumed here that we are dealing with a 1-stage reducible system and not a higher-reducible one. This means that the functions $Z_{a_{1}}^{a}$ have no zero modes themselves.

\footnotetext{
${ }^{3}$ For the exact regularity conditions on the action and symmetries such that eq. (3.20) can be demanded, see e.g. $[28,14]$

${ }^{4}$ The $\psi$ can be taken out of this equation, but writing it in this way avoids a number of minus signs.
} 
From the bracket of eq.(3.19) with $Z_{b_{1}}^{b}$ and using eq.(3.21) one can derive that

$$
T_{a}\left\{\left[Z_{a_{1}}^{a}, Z_{b_{1}}^{b}\right]+\left[Z_{b_{1}}^{a}, Z_{a_{1}}^{b}\right](-)^{\left(a_{1}+b\right) b_{1}+b a_{1}}\right\}=0 .
$$

One could use eq.(3.20) to constrain the expression in brackets but we assume further that it is vanishing, as it is the case in the DISP action (see below).

For gauge fixing we can introduce again a non-minimal sector and a gauge fermion containing anti-ghosts, extra ghosts and Lagrange multipliers

$$
\begin{aligned}
S^{n m} & =b^{* a} \lambda_{a}+\check{b}^{* a_{1}} \check{\lambda}_{a_{1}}+b^{* a_{1}} \lambda_{a_{1}}+b_{a_{1}}^{\prime *} \lambda^{\prime a_{1}} \\
\Psi & =b_{a} \psi^{a}+b_{a_{1}} \check{c}^{a_{1}}+b^{\prime a_{1}} \check{b}_{a_{1}} .
\end{aligned}
$$

We can visualise this in the following scheme

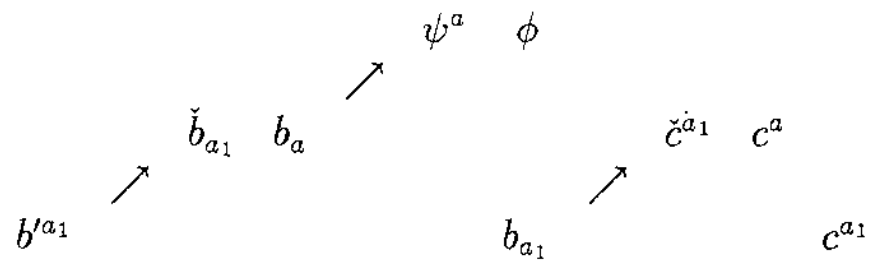

(the arrows represent the terms in the gauge fermion). The Lagrange multipliers form a second table, which we omit here. Using our lemma B.3, this implies that the system $\left\{\tilde{b}_{a_{1}}, \lambda_{a_{1}}, b^{\prime a_{1}}, \lambda^{\prime a_{1}}\right\}$ has become trivial and that one should replace in the extended action

$$
\begin{aligned}
& \psi^{a} \rightarrow-b^{* a} \\
& \psi_{a}^{*} \rightarrow b_{a} \\
& \check{c}^{a_{1}} \rightarrow-b^{* a_{1}} \\
& \check{c}_{a_{1}}^{*} \rightarrow b_{a_{1}} .
\end{aligned}
$$

Thus we can conclude that the Lagrange multipliers disappear, together with the antighosts and extra ghosts which are not connected with arrows to the minimal ghost sector in (3.25). On the other hand the antighosts which are connected to the minimal sector replace the corresponding ghosts, and instead of introducing the whole non-minimal sector we could just have performed these canonical transformations given by eq.(3.26). This leads to the following result:

$$
\begin{aligned}
S_{g f}^{1 r e d}= & S_{g f}^{i r r}-b_{a} Z_{a_{1}}^{a} b^{* a_{1}}-c_{a}^{*} Z_{a_{1}} c^{a_{1}} \\
& +b_{a_{1}} \dot{c}^{a_{1}}+b_{a} \phi_{i}^{*}\left[\phi^{i}, Z^{a}{ }_{a_{1}}\right] c^{a_{1}}(-)^{a} .
\end{aligned}
$$

$S_{g f}^{i r r}$ is given in eq.(3.13). Following eq.(3.2) the BRST operator is then given by

$$
\Omega=T_{a} c^{a}+\frac{1}{2} b_{a} f_{b c}^{a} c^{c} c^{b}(-)^{b}-b_{a} Z_{a_{1}}^{a} c^{a_{1}}
$$

As described for the irreducible case we can also go the other way around. Starting from the BRST operator one can apply the same steps as in the irreducible case and one would then end up with the extended action given in eq.(3.22).

We now proceed with the infinite reducible case. 


\subsection{First-order action Lagrangian quantisation for infinite-reducible systems}

The difference with the 1-stage reducible case is that there are now also at the next second-order level zero mode matrices, i.e. $Z_{a_{1}}^{a} Z_{a_{2}}^{a_{1}} \sim T_{b}$. These second-order zero mode matrices have two implications. First of all they lead to additional first-order zero modes in the Lagrangian. We will use ghosts $\breve{c}^{a_{2}}$ for them. Secondly, they imply second order zero modes for which we introduce ghosts $c^{a_{2}}$. This is completely analogous to what we discussed above for the first-order zero mode matrices $Z^{a}{ }_{a_{1}}$. In the infinite reducible case this repeats itself at all the next levels [29]. We thus get the following scheme of (minimal) ghosts :

\begin{tabular}{c|cccc} 
ghost number & field & statistics & field & statistics \\
\hline 1 & $c^{a}$ & $(-)^{a+1}$ & $\check{c}^{a_{1}}$ & $(-)^{a_{1}+1}$ \\
2 & $c^{a_{1}}$ & $(-)^{a_{1}}$ & $\check{c}^{a_{2}}$ & $(-)^{a_{2}}$ \\
$\vdots$ & & $\vdots$ & & \\
$\mathrm{p}$ & $c^{a_{p-1}}$ & $(-)^{a_{p-1}+p}$ & $\check{c}^{a_{p}}$ & $(-)^{a_{p}+p}$ \\
$\vdots$ & & $\vdots$ & &
\end{tabular}

We have seen above that we can go as well from a classical action with gauge invariances to a gauge fixed action with a BRST operator as in the inverse way. In the infinite-reducible case the BRST operator is in general simpler. We will therefore in this case start from eq.(3.2) with the BRST operator and rederive the classical action with its gauge invariances.

Motivated by the form of the BRST operator eq.(3.3) we consider a BRST operator (which should have ghost number 1) with fields $\phi$ of ghost number $0, c^{a_{p}}$ of ghost number $p+1$ and $b_{a_{p}}$ of ghost number $-p-1$ (with $c^{a_{0}} \equiv$ $\left.c^{a}, b_{a_{0}} \equiv b_{\alpha}\right)$. We assume that the only terms with more than two fields of non-vanishing ghost number are trilinear terms containing an antighost of ghost number -1 .

Our starting point is thus the following BRST operator:

$$
\Omega=T_{a}(\phi) c^{a}+\frac{1}{2} b_{a} f_{b c}^{a} c^{c} c^{b}(-)^{b}-b_{a_{p}} Z_{a_{p+1}}^{a_{p}}(\phi) c^{a_{p+1}}+b_{a} b_{a_{p}} f_{a_{p+2}}^{a_{p}{ }_{a}} c^{a_{p+2}} .
$$

We assume a sum over $p=0,1, \ldots$. The index $a_{0}$ is the same as the index $a$ which we used in the previous cases. The functions in the last two terms are defined below. The ghosts and antighosts $b_{a_{p}}$ and $c^{a_{p}}$ have statistics $a_{p}+p+1$, and the functions $f^{a_{p} a}{ }_{a_{2}}$ satisfy the symmetry property

$$
f^{a_{p} a}{ }_{a_{p+2}}=(-)^{(a+1)\left(a_{p}+p+1\right)} f^{a a_{p}}{ }_{a_{p+2}} .
$$

We will now give the conditions on the coefficients and functions in eq.(3.29) in order that this operator is nilpotent, and then derive a classical action of all the ghost number zero fields of which this can be seen as the gauge fixed BRST operator. 
One can check that the BRST operator given above is nilpotent provided that the following conditions hold:

1. $\left[T_{a}, T_{b}\right]=T_{c} f_{a b}^{c}$, where we assume that the $f$ are constants and satisfy the Jacobi identities (i.e. without using zero modes).

2. The zero modes of $T_{a}$ are defined by $T_{a} Z^{a}{ }_{a_{1}}=0$ and satisfy $f^{a}{ }_{c b} \psi^{b} Z_{a_{1}}^{c}-\left[T_{b} \psi^{b}, Z_{a_{1}}^{a}\right]=0$.

3. The following zero modes define also the coefficients in the last term of $\Omega$ :

$$
\begin{aligned}
& Z_{a_{1}}^{a} Z_{a_{2}}^{a_{1}}=2(-)^{a} T_{b} f_{a_{2}}^{b a} \\
& Z_{a_{p+1}}^{a_{p}} Z_{a_{p+1}}^{a_{p+1}}=(-)^{a_{p}+p} T_{a} f^{a a_{p}}{ }_{a_{p+2}} \text { for } p \geq 1 .
\end{aligned}
$$

Again these coefficients $f$ are assumed to be constants. We assume also that these higher zero modes have vanishing brackets with the currents :

$$
\left[Z^{a_{p}} a_{p+1}, T_{a}\right]=0 \text { for } p \geq 1 \text {. }
$$

4. $b_{a} b_{a_{p}} f^{a_{p} a}{ }_{a_{p+2}} b_{b} f^{b a_{p+2} a_{p+4}}=0$

5. $b_{a} b_{a_{p}}\left(Z^{a_{p}}{ }_{a_{p+1}} f^{a_{p+1} a_{a_{p+3}}}(-)^{a+1}+f^{a_{p} a}{ }_{a_{p+2}} Z^{a_{p+2}}{ }_{a_{p+3}}\right)=0$

6. $b_{d} f_{a e}^{d} c^{e}(-)^{a} b_{a_{p}} f^{a_{p} a}{ }_{a_{p+2}}=0$

7. $\left[Z^{a_{p}}{ }_{a_{p+1}}, Z^{b_{q_{q}}} b_{q+1}\right]=0$ if $p \neq q$

8. $\left[Z^{a_{p} a_{p+1}}, Z^{b_{p}} b_{p+1}\right]+\left[Z^{a_{p}} b_{p+1}, Z^{b_{p}} a_{p+1}\right](-)^{b_{p}\left(a_{p+1}+b_{p+1}\right)+b_{p+1} a_{p+1}+p}=0$.

The last equation was already obtained for $p=0$ in the 1-stage reducible case. As we wrote there, the expression is in general already constrained due to previous equations. The same remark also holds for the previous equations (e.g. one can derive that $T_{a} \frac{\partial_{\ell}}{\partial b_{a}}$ on the fifth equation follows from $Z Z Z$ in two different ways using eq. (3.31)). If these conditions would not be satisfied we should introduce more terms in eq.(3.29).

From eq.(3.29) we now derive an extended action as follows. We first use eq.(3.2), as we did in the irreducible case. We then undo the gauge fixings by applying the following generalisation of eq.(3.16) to the infinite reducible case :

$$
\begin{array}{rlrl}
b^{* a} & \rightarrow & -\psi^{a} ; & b_{a} \rightarrow \psi_{a}^{*} \\
b^{* a_{p+1}} & \rightarrow-\check{c}^{a_{p+1}} ; & b_{a_{p+1}} \rightarrow \check{c}_{a_{p+1}}^{*}
\end{array}
$$

Note that these replacements conserve also the ghost number as $g h\left(b_{a_{p}}\right)=$ $-p-1$ and eq.(A.3). The procedure to go between the gauge fixed action and the classical action amounts thus to change the negative ghost number fields ('antighosts') by the fields in the second column of the table in the 
beginning of this section. The latter are identified with the antifields of the antighosts.

Making these substitutions in the BRST operator gives rise to a fermion

$$
\begin{aligned}
\Omega^{\prime}= & T_{a}(\phi) c^{a}+\frac{1}{2} \psi_{a}^{*} f_{b c}^{a} c^{c} c^{b}(-)^{b}-\psi_{a}^{*} Z_{a_{1}}^{a}(\phi) c^{a_{1}} \\
& -\tilde{c}_{a_{p+1}}^{*} Z^{a_{p+1}}{ }_{a_{p+2}} c^{a_{p+2}}+\psi_{a}^{*} \psi_{b}^{*} f^{b a}{ }_{a 2} c^{a_{2}}+\psi_{a}^{*} \tilde{c}_{a_{p+1}}^{*} f^{a_{p+1}{ }_{a_{p+3}}} c^{a_{p+3}} .
\end{aligned}
$$

This gives then rise to the following extended action before gauge fixing:

$$
\begin{aligned}
S= & \frac{1}{2} \phi^{i} C_{i j} \dot{\phi}^{j}-H_{0}+\psi_{a}^{*} \dot{c}^{a}+\check{c}_{a_{p+1}}^{*} \dot{c}^{a_{p+1}} \\
& +\phi_{i}^{*}\left[\phi^{i}, \Omega^{\prime}\right]+c_{a}^{*} \frac{\partial_{l}}{\partial \psi_{a}^{*}} \Omega^{\prime}+c_{a_{p+1}}^{*} \frac{\partial_{l}}{\partial \check{c}_{a_{p+1}}^{*}} \Omega^{\prime}-\frac{\partial_{r}}{\partial c^{a}} \Omega^{\prime} \psi^{a}-\frac{\partial_{r}}{\partial c^{a_{p+1}}} \Omega^{\prime} \tilde{c}^{a_{p+1}} .
\end{aligned}
$$

So this is the extension of eq.(3.22) to an arbitrary reducibility level. We should stress that this extended action is just the action eq.(3.2) after the canonical transformation eq.(3.33).

For further use we give now the explicit expression for this extended action

$$
\begin{aligned}
S_{C}= & \frac{1}{2} \phi^{i} C_{i j} \dot{\phi}^{j}-H_{0}-T_{a} \psi^{a} \\
& +\phi_{i}^{*}\left[\phi^{i}, T_{a}\right] c^{a}+\psi_{a}^{*}\left(\dot{c}^{a}+f^{a}{ }_{b c} \psi^{c} c^{b}+Z_{a_{1}}^{a} \check{c}^{a_{1}}\right)+\psi_{a}^{*} \phi_{i}^{*}\left[\phi^{i}, Z^{a}{ }_{a_{1}}\right] c^{a_{1}}(-)^{a} \\
& +\frac{1}{2} c_{a}^{*} f^{a}{ }_{b c} c^{c} c^{b}(-)^{b}+\check{c}_{a_{p+1}}^{*}\left(\dot{c}^{a_{p+1}}-Z^{a_{p+1}}{ }_{a_{p+2}} \check{c}^{a_{p+2}}\right)-c_{a_{p}}^{*} Z^{a_{p}{ }_{a_{p+1}} c^{a_{p+1}}} \\
& +2 c_{a}^{*} \psi_{b}^{*} f_{a_{2}}^{b a} c^{a_{2}}+\left(c_{a}^{*} \check{c}_{a_{p+1}^{*}}-(-)^{a} \psi_{a}^{*} c_{a_{p+1}}^{*}\right) f^{a_{p+1}{ }_{a_{p+3}} c^{a_{p+3}}} \\
& -\psi_{a}^{*} \psi_{b}^{*} f_{a_{2}}^{b a} \check{c}^{a_{2}}-\psi_{a}^{*} \check{c}_{a_{p+1}}^{*} f^{a_{p+1} a}{ }_{a_{p+3}} \check{c}^{a_{p+3}}
\end{aligned}
$$

where the variable $p$ is always defined such that sums over $p$ range from 0 to $\infty$. We have thus shown that this classical action with gauge invariances can be gauge fixed to a form eq.(3.2) with BRST operator eq.(3.29) and a matrix $C_{I J}$ such that in the ghost sector

$$
\left[c^{a_{p}}, b_{b_{q}}\right]=\delta_{b}^{a} \delta_{q}^{p} .
$$

\subsection{Application to the DISP action}

The general procedure in the previous subsection has been set up such that it is directly applicable to the DISP action and BRST operator. We just have to make a translation table for the fields. The classical DISP superparticle action of [11] eq.(3.4), and the BRST operator [10] eq.(3.3) with gauge fixed action eq.(3.1) are of the general form given resp. in eq.(3.9) (with the gauge invariances in eq.(3.36)) and in eq.(3.29) and eq.(3.2) with the following identifications. For the ghost number zero fields ${ }^{5}$

$$
\left\{\phi^{i}\right\}=\left\{X^{\mu}, P_{\mu}, \theta_{p} \equiv \theta_{p, p}=\theta(p, 0), \lambda^{p} \equiv \bar{\lambda}^{p, p}=\lambda(p, 0)\right\}
$$

\footnotetext{
${ }^{5}$ To facilitate the comparison with [11] we give here also the notation of the fields which was used there. The labels refer then to $S U(2)$ representations, e.g. $\theta(j, m)$
} 
and $H_{0}=\frac{1}{2} P^{2}$. We have then the brackets

$$
\left[X^{\mu}, P_{\nu}\right]=\delta_{\nu}^{\mu} ; \quad\left[\theta_{p}, \lambda^{q}\right]=\delta_{p}^{q} .
$$

The currents and Lagrange multipliers are given by

$$
\begin{aligned}
T_{a} & =\left\{\frac{1}{2} P^{2}, \quad K_{p}, \quad F^{p}\right\} \\
\psi^{a} & =\left\{g-1, \quad \zeta^{p}, \quad \eta_{p}\right\} .
\end{aligned}
$$

Here we are using the following notations:

$$
\begin{aligned}
& K_{p}=\lambda^{p} \not p \\
& F^{p}=\lambda^{p+1}-\not p \theta_{p+1}+\lambda^{p}+\not p \theta_{p} .
\end{aligned}
$$

Note that the only non-trivial part of the superalgebra

$$
\left[T_{a}, T_{b}\right]=T_{c} f_{a b}^{c}
$$

is

$$
\left[K_{q}, \quad F^{p}\right]=-P^{2}\left(\delta_{q}^{p+1}-\delta_{q}^{p}\right)
$$

For the first ghosts and antighosts we have

$$
\begin{aligned}
& c^{a}=\left\{c ; \quad \theta_{p+1, p}=\theta\left(p+\frac{1}{2}, \frac{1}{2}\right) ; \lambda_{p+1, p+1}=\lambda\left(p+\frac{1}{2}, \frac{1}{2}\right)\right\} \\
& b_{a}=\left\{b ; \quad \bar{\lambda}^{p+1, p}=\lambda\left(p+\frac{1}{2},-\frac{1}{2}\right) ;-\bar{\theta}^{p+1, p+1}=-\theta\left(p+\frac{1}{2},-\frac{1}{2}\right)\right\}(.3 .4
\end{aligned}
$$

In the following we denote the values of the index $a$ as $\bullet$ for the first constraint, $q$ for the second infinite series of constraints and $q^{\prime}$ for the last infinite series. Using the above expressions for $T_{a}$ we find that there are two infinite series of zero modes, which we will label by $q_{1}$ and $q_{1}^{\prime}$. Together they form the index $a_{1}$ from above. The same pattern repeats itself at each stage such that $a_{2}=\left(q_{2}, q_{2}^{\prime}\right), a_{3}=\left(q_{3}, q_{3}^{\prime}\right), \ldots$. All these indices except $\bullet$ are fermionic : $(-)^{a_{p}}=-$ for $p>0$ and for $p=0$ if $a_{0} \neq \bullet$. We find that the first stage zero mode matrix is given by

$$
Z_{a_{1}}^{a}=\left(\begin{array}{cc}
Z_{p}=-2 \lambda^{p} ; & Z_{p^{\prime}}=-2\left(\theta_{p^{\prime}}-\theta_{p^{\prime}+1}\right) \\
Z_{p}^{q}=\delta_{p}^{q} \not P ; & Z_{p^{\prime}}^{q}=-\delta_{p^{\prime}}^{q}-\delta_{p^{\prime}+1}^{q} \\
Z^{q^{\prime}}=0 ; & Z^{q^{\prime}}{ }_{p^{\prime}}=\delta_{p^{\prime}}^{q^{\prime}} \not p
\end{array}\right) .
$$

This has further zero modes (for $p \geq 1$ )

$$
Z_{a_{p+1}}^{a_{p}}=\left(\begin{array}{cc}
Z_{r_{p+1}}^{q_{p}}=\delta_{r}^{q} \not p ; & Z_{r_{p+1}}^{q_{p}}=(-)^{p+1}\left(\delta_{r^{\prime}}^{q}+\delta_{r^{\prime}+1}^{q}\right) \\
Z_{r_{p+1}}^{q_{p}}=0 ; & Z_{r_{p+1}^{q_{p}}}^{q_{r_{p}}^{\prime}}=\delta_{p^{\prime}}^{q^{\prime} \not p}
\end{array}\right) .
$$

Further we find the following non-zero coefficients

$$
\begin{aligned}
& f_{q_{2}}^{p \bullet}=f_{q_{2}}^{\bullet p}=-\delta_{q}^{p} ; \quad f_{q_{2}^{\prime}}^{p^{\prime}}=f_{q_{2}^{\prime}}^{\bullet p^{\prime}}=-\delta_{q^{\prime}}^{p^{\prime}} \\
& f^{q_{p} \bullet}{ }_{{ }_{p+2}}=-2 \delta_{r}^{q} ; \quad f^{q_{p}^{\prime} \bullet}{ }_{r_{p+2}^{\prime}}=-2 \delta_{r^{\prime}}^{q^{\prime}} \text { for } p \geq 1 .
\end{aligned}
$$


One can then check that all the conditions of the previous subsection are satisfied.

Finally, we make the identifications:

$$
\begin{aligned}
& c^{q_{p}}=\theta_{q+p+1, q}(-)^{p}=\theta\left(q+\frac{p+1}{2}, \frac{p+1}{2}\right)(-)^{p} \\
& c^{q_{p}^{\prime}}=\lambda_{q+p+1, q+1}(-)^{\frac{p(p-1)}{2}}=\lambda\left(q+\frac{p+1}{2}, \frac{p+1}{2}\right)(-)^{\frac{p(p-1)}{2}} \\
& b_{q_{p}}=\bar{\lambda}^{q+p+1, q}(-)^{p}=\lambda\left(q+\frac{p+1}{2},-\frac{p+1}{2}\right)(-)^{p} \\
& b_{q_{p}^{\prime}}=-\bar{\theta}^{q^{\prime}+p+1, q^{\prime}+1}(-)^{\frac{p(p+1)}{2}}=-\theta\left(q+\frac{p+1}{2},-\frac{p+1}{2}\right)(-)^{\frac{p(p+1)}{2}} .
\end{aligned}
$$

The former two have ghost number $p+1$, the latter two have ghost number $-(p+1)$. The statistics is now always $(-)^{p}$.

This finishes the identifications of the fields. Using the previous subsection we have thus proved that the gauge fixed action and DISP-BRST operator [10] are related by canonical transformations to the classical DISP action [11]. In the beginning of the next section we will insert eqs. $(3.44)-(3.48)$ in eq.(3.36) to re-obtain the classical DISP action eq.(3.4) with its gauge transformations and zero modes. Also one can check that inserting these same equations in eq.(3.29) gives rise to the DISP-BRST operator eq.(3.3). One remarks the great simplification which we have obtained by recognizing the role of the fields in the DISP picture.

\section{Relation between the DISP and the GS action}

Let us start by writing here the complete extended DISP action. It follows from eq.(3.36) by using the identifications from subsection 3.4.

$$
\begin{aligned}
S= & P \dot{X}-\frac{1}{2} g P^{2}+\lambda^{p} \dot{\theta}_{p}-\lambda^{p} \not \not \zeta^{p}-\left\{\lambda^{p+1}-\theta_{p+1} \not P+\lambda^{p}+\theta_{p} \not p\right\} \eta_{p} \\
& +X_{\mu}^{*}\left(\lambda^{p} \gamma^{\mu} c^{p}+\theta_{p} \gamma^{\mu}\left(c^{p^{\prime}}-c^{p^{\prime}-1}\right)+P^{\mu} c\right) \\
& +\theta^{* p}\left(\not P c^{p}+c^{p^{\prime}}+c^{p^{\prime}-1}\right) \\
& +\zeta_{p}^{*}\left(\dot{c}^{p}+\not p \check{c}^{p_{1}}-\check{c}^{p_{1}^{\prime}}-\check{c}^{p_{1}^{\prime}-1}\right) \\
& +g^{*}\left(\dot{c}+\eta_{p}\left(c^{p}-c^{p+1}\right)+2 \zeta^{p}\left(c^{p^{\prime}}-c^{p^{\prime}-1}\right)-2 \check{c}^{p_{1}^{\prime}}\left(\theta_{p}-\theta_{p+1}\right)-2 \lambda^{p^{p}} \check{c}^{p_{1}}\right) \\
& +\zeta_{p}^{*}\left(\dot{c}^{p}+\not \not \check{c}^{p_{1}}-\check{c}^{p_{1}^{\prime}}-\check{c}^{p_{1}^{\prime}-1}\right) \\
& +\eta_{p}^{*}\left(\dot{c}^{p^{\prime}}+\not P \check{c}^{p_{1}^{\prime}}\right) \\
& -2 g^{*} \theta_{p}^{*} c^{p_{1}}+2 g^{*}\left(\lambda_{p+1}^{*}-\lambda_{p}^{*}\right) c^{p^{\prime}}-\left(\zeta_{p}^{*}+\eta_{p}^{*}\right) X_{\mu}^{*} \gamma^{\mu} c^{p_{1}} \\
& +2 c^{*}\left(c^{p^{\prime}}\left(c^{p+1}-c^{p}\right)+\left(\theta_{p}-\theta_{p+1}\right) c^{p_{1}^{\prime}}-\not p \theta_{p} c^{p_{1}}\right)
\end{aligned}
$$




$$
\begin{aligned}
& +c_{q_{p}}^{*}\left(-\not \not c^{q_{p+1}}+c^{q_{p+1}^{\prime}}+c^{q_{p+1}^{\prime}-1}\right)-c_{q_{p}^{\prime}}^{*} \not \not c^{q_{p+1}^{\prime}} \\
& +\check{c}_{q_{p+1}}^{*}\left(\dot{c}^{q_{p+1}}-\not \not \check{c}^{q_{p+2}}+\left(\check{c}^{q_{p+2}}+\check{c}^{q_{p+2}-1}\right)\right) \\
& +\check{c}_{q_{p+1}^{\prime}}^{*}\left(\dot{c}^{q_{p+1}^{\prime}}-\not \not \check{c}^{q_{p+2}^{\prime}}\right) \\
& +2 c_{a}^{*} \psi_{b}^{*} f_{a_{2}}^{b a} c^{a_{2}}+\left(c_{a}^{*} \check{c}_{a_{p+1}}^{*}-(-)^{a} \psi_{a}^{*} c_{a_{p+1}}^{*}\right) f^{a_{p+1} a}{ }_{a_{p+3}} c^{a_{p+3}} \\
& -\psi_{a}^{*} \psi_{b}^{*} f_{a_{2}}^{b a} \check{c}^{a_{2}}-\psi_{a}^{*} \check{c}_{a_{p+1}}^{*} f^{a_{p+1} a}{ }_{a_{p+3}} \check{c}^{a_{p+3}} .
\end{aligned}
$$

Note the summation conventions: when an index is repeated as in $\theta_{p} c^{p^{\prime}}$ or $\zeta_{p}^{*} \check{c}^{p_{1}}$ it is also summed over $p$ (always from 0 to $\infty$ ). The extra indications' or 1 of indices indicate only the type of ghost field.

The questions which can be posed at this point are the following. The BS and the DISP action describe the same physical states. Is there a canonical transformation between their extended actions? Can one gauge fix the BS action by canonical transformations to obtain the gauge fixed action with the DISP-BRST operator ?

In [11] a partial gauge fixing was given to obtain the classical BS action from the classical DISP action. If this procedure can be performed by a canonical transformation, then one seems to have an answer to the previous questions. Indeed then the combination of the inverse canonical transformation, bringing us back to the classical DISP action, together with the canonical transformations which were needed to perform the gauge fixing of the DISP action, would achieve a gauge fixing to the BS action obtaining the desired BRST operator. Therefore we now look to the mentioned gauge fixing in [11] and perform it using only canonical transformations.

We begin by adding the non-minimal system $S_{n m}=b^{* p} \pi_{p}$ and choose the following gauge fermion:

$$
\Psi=b_{p}\left(\lambda^{p}+\not p \theta_{p}-\lambda^{p+1}+\not p \theta_{p+1}\right) .
$$

The field equations of $\pi_{p}$ and the Lagrange multiplier $\eta_{p}$ put $\lambda^{p}$ equal to - $P \theta_{p}$. To do this including the full extended action we will apply our lemma B.1. Therefore we first diagonalise the action by performing canonical transformations on $\lambda^{p}, \theta_{p}, \eta_{p}, \pi_{p}$. We want to define

$$
\begin{aligned}
\tilde{\lambda}^{p} & =\lambda^{p}+\not p \theta_{p} \\
\eta_{ \pm, p} & =\eta_{p} \pm \pi_{p} .
\end{aligned}
$$

The first line is in fact the variable $\tilde{Q}^{p}$ of [11]. This transformation is obtained by a fermionic generating function

$$
F=\tilde{\lambda}_{p}^{*}\left(\lambda^{p}+\not p \theta_{p}\right)+\tilde{\theta}^{* p} \theta_{p}+\eta_{+}^{* p}\left(\eta_{p}+\pi_{p}\right)+\eta_{-}^{* p}\left(\eta_{p}-\pi_{p}\right)+\ldots
$$

where the ... refer to terms which generate a unit transformation on the other variables (terms of the form $\tilde{\Phi}_{I}^{*} \Phi^{I}$ ). We rename $\eta_{+, p}$ to $\eta_{p}$. Then we redefine $g$, absorbing everything proportional to $P^{2}$, and in the same way we absorb all terms proportional to $\not p \theta_{p+1}$ in $\eta_{p}$. 
Having performed all these manipulations we obtain

$$
\begin{aligned}
& S_{C}=P \dot{X}-\theta \not P \dot{\theta}-\frac{1}{2} g P^{2} \\
& +4 b_{p} \not P \lambda_{p+1, p+1}+2 \theta_{p+1} \not P \eta_{p} \\
& +X_{\mu}^{*}\left(P^{\mu} c+\theta \gamma^{\mu} \not P \theta_{1,0}\right) \\
& +g^{*}\left(\dot{c}+4 \dot{\theta} \theta_{1,0}\right)-2 c^{*} \theta_{1,0} \not P \theta_{1,0} \\
& +\sum_{p=q}^{\infty} \sum_{q=0}^{\infty}\left[\theta_{p, q}^{*} \not p \theta_{p+1, q}+(-)^{\frac{1}{2}(p-q)(p-q-1)}\left(\theta_{p, q}^{*}+\theta_{p+1, q+1}^{*}\right) \lambda_{p+1, q+1}\right. \\
& \left.-(-)^{(p-q)} \lambda_{p+1, q+1}^{*} \not / \lambda_{p+2, q+1}\right] \\
& +X_{\mu}^{*}\left(\theta_{p}-\theta_{p+1}\right) \gamma^{\mu} \lambda_{p+1, p+1} \\
& +\frac{1}{2} b^{* p}\left(\frac{1}{2} \dot{\theta}_{p+1}-\frac{1}{2} \dot{\theta}_{p}+\not p \zeta_{p}+\eta_{p}+\eta_{p-1}\right)-\frac{1}{4} b^{* 0} \dot{\theta} \\
& +\zeta_{p}^{*}\left(\dot{\theta}_{p+1, p}+\not p \check{c}^{p_{1}}-\check{c}^{p_{1}^{\prime}}-\check{c}^{p_{1}^{\prime}-1}\right) \\
& +\eta_{p}^{*}\left(\not \check{c}^{p_{1}^{\prime}}+\frac{1}{2}\left(\dot{\lambda}_{p+1, p+1}-\dot{\lambda}_{p+2, p+2}-\not P \dot{\theta}_{p+2, p+1}\right)\right) \\
& +2 c^{*}\left(\left(\lambda_{p+1, p+1}-\lambda_{p+2, p+2}\right) \theta_{p+2, p+1}-2 \lambda_{1,1} \theta_{1,0}+\theta_{p+1}\left(\lambda_{p+3, p+2}-\lambda_{p+2, p+1}\right)\right) \\
& +\check{c}_{a_{p+1}^{*}}^{*}\left(\dot{c}^{a_{p+1}}-Z^{a_{p+1}{ }_{a_{p+2}}} \check{c}^{a_{p+2}}\right) \\
& +g^{*}\left(-4 \eta_{p} \theta_{p+2, p+1}+4 \zeta^{p} \lambda_{p+1, p+1}-8 b_{p} \lambda_{p+2, p+1}+4 \check{c}^{p_{1}^{\prime}} \theta_{p+1}-2 \theta_{p+1} \dot{\theta}_{p+2, p+1}\right) \\
& +2 g^{*} \sum_{p=q}^{\infty} \sum_{q=0}^{\infty}\left[\theta_{p, q}^{*} \theta_{p+2, q}-\lambda_{p+1, q+1}^{*} \lambda_{p+3, q+1}\right] \\
& +2 g^{*} X_{\mu}^{*}\left(\theta_{p, p} \gamma^{\mu} \theta_{p+2, p}-\theta_{1,0} \gamma^{\mu} \theta_{1,0}\right) \\
& +4 g^{*} c^{*}\left(-\theta_{1,0} \theta_{2,0}+\theta_{p, p} \theta_{p+3, p}\right) \\
& +2 c^{*} \psi_{a}^{*} f_{a_{2}}^{a^{\bullet}} c^{a_{2}}+c^{*} \check{c}_{a_{p+1}^{*}}^{*} f^{a_{p+1} \bullet}{ }_{a_{p+3}} c^{a_{p+3}} \\
& +\frac{1}{2} b^{* p}\left(\frac{1}{2} X_{\mu}^{*} \gamma^{\mu}\left(\theta_{p+1, p}-\theta_{p+2, p+1}\right)-2 g^{*} \check{c}^{p_{1}}+c^{*}\left(\theta_{p+2, p}-\theta_{p+3, p+1}\right)\right) \\
& +\frac{1}{2} b^{* 0}\left(\frac{1}{2} X_{\mu}^{*} \gamma^{\mu} \theta_{1,0}+c^{*} \theta_{2,0}\right) \\
& +\left(\zeta_{p}^{*}+\eta_{p}^{*}\right) X_{\mu}^{*} \gamma^{\mu} \theta_{p+2, p} \\
& -2 g^{*} \psi_{a}^{*} f_{a_{2}}^{a^{\bullet}} \check{c}^{a_{2}}-g^{*} \check{c}_{a_{p+1}^{*}}^{*} f^{a_{p+1} \bullet}{ }_{a_{p+3}} \check{c}^{a_{p+3}} \\
& -\eta_{p}^{*} X_{\mu}^{*}\left(P^{\mu} \theta_{p+3, p+1}-\gamma^{\mu} \theta_{p+2, p}\right) \\
& +g^{*} \eta_{p}^{*}\left(-\dot{\theta}_{p+3, p+1}+X_{\mu}^{*} \gamma^{\mu} \theta_{p+4, p+1}+2 c^{*} \theta_{p+5, p+1}\right)
\end{aligned}
$$

We have achieved our goal. The reader can recognise the first line as the classical BS superparticle action and the lines 3,4 and some terms below as the antifield dependent parts of eq.(2.3). The remaining two terms in the antifield independent part of eq.(4.5) do not lead to extra propagating fields in the field equations. Therefore we expect that this action describes the same physics as the BS action. However, it cannot be seen as a classical action as it contains fields of non-zero ghost number. 
We see no regular canonical transformations to bring this extended action to the BS form plus possible trivial terms. This means that we found that the 'partial gauge fixing' does not completely lead to the BS extended action if we use only canonical transformations. We conclude that we do not have a canonical transformation between the BS and DISP extended actions, although their classical theories describe the same number of physical variables. We cannot gauge fix the $\mathrm{BS}$ action to the gauge fixed action with the DISP-BRST operator by performing canonical transformations on their extended action and adding or subtracting trivial systems.

\section{Conclusions}

We have proved in this paper that there is a clear gauge fixing procedure for the classical DISP action introduced in [11] to obtain the gauge fixed action with BRST operator given in [10]. Moreover we found the procedure, based on canonical transformations in the space of fields and antifields, to go back from this gauge fixed action to the classical action. This procedure is in fact general for first-order actions.

The fields which we use are the infinite tower of ghosts, antighosts, extra ghosts and Lagrange multipliers introduced in the BV scheme for quantisation of the BS action. However, the procedure mentioned above makes a re-interpretation of all these fields. We start from a gauge fixed action with fields of all ghost numbers. We then change all the negative ghost number fields with their antifields. If $g$ is the negative ghost number of such a field, then its antifield has ghost number $-g-1 \geq 0$ and will be interpreted as a field or minimal ghost in the extended action corresponding to the classical theory. So e.g. all ghost number zero fields of the gauge fixed action (which contained in the BS case an infinite series of extra ghosts) are interpreted as classical fields. Also the ghost number -1 fields (or better, their antifields) are replaced by classical gauge fields, multiplying the constraints generating the gauge transformations (as e.g. $b^{*} \rightarrow-g$, a procedure known from the proofs of equivalence between Lagrangian and Hamiltonian quantisations [27] and from Fujikawa regularisation [30]). All ghost number 1 fields become ghosts, as well as the antifields of the ghost number -2 fields (ghosts of symmetries due to zero modes of constraints). Ghost number 2 and -3 become ghosts for ghosts, ... .

We had already shown in [10] that the DISP-BRST operator has the correct physical states as solutions of its cohomology. As we performed only canonical transformations, the DISP classical action also describes these physical states, and in this paper we gave the proof explicitly for this classical action. On the other hand we found no canonical transformations for going to the BS action. Nevertheless the classical BS action and the classical DISP action describe the same physical states. In section 2.1 we performed a procedure to obtain a gauge fixed BS action with BRST operator, but the 
latter had extra solutions of its cohomology equations. We will now explicitly indicate from where these extra solutions come.

We first note that the antibracket cohomology of eq.(2.8) is still the right one. The solutions are the same as in eq.(2.4), with the substitution $g^{*} \rightarrow b$. Then we performed the fixing of the Stueckelberg symmetry and obtained the extended action given in eq.(2.11). It has the previous solutions with some modifications due to canonical transformations

$$
\begin{aligned}
P^{\mu} & \equiv X^{\mu}-\frac{P^{\mu}}{P^{+}} X^{+}-\frac{1}{2} \frac{P^{\nu}}{P^{+}} \theta \gamma^{+\mu \nu} \theta-2 b \frac{1}{P^{+}} \theta \gamma^{+\mu} \theta_{1,0} \\
-\frac{1}{2} \sum_{p=0}^{\infty} \frac{1}{P^{+}} \bar{\lambda}^{p, 0} \gamma^{\mu+} \theta_{p, 0} & \\
s^{0,0} & \equiv \not P \theta-2 b \theta_{1,0} .
\end{aligned}
$$

However, we now got an extra solution

$$
\mathcal{S} \bar{\lambda}^{0,0}=0
$$

with

$$
\mathcal{S} \bar{\lambda}^{1,0}=\not \partial \bar{\lambda}^{0,0}
$$

Before the last step we had $\mathcal{S} \bar{\theta}^{0,0}=\bar{\lambda}^{0,0}$. The elimination of $\bar{\theta}^{0,0}$ thus produced this new solution of the cohomology, which then remained in the BRST cohomology.

We have illustrated the above remarks in appendix $\mathrm{C}$. We gave there a model where also such an extra solution was created due to an analogous 'second step gauge fixing procedure' and we also showed there which second step gauge fixing procedures are allowed. From the example there, one gets a suggestion how to continue in the BS case. It consists of adding a trivial nonminimal sector, which after an elimination of auxiliary fields as in lemma B.3, consists of interchanging the $\bar{\theta}^{p, 0}$ with $\left(\bar{\theta}^{p, 0}\right)^{*}$. If we do this, we can eliminate the new field $\bar{\theta}^{p, 0}$ (former antifield) together with $\bar{\lambda}^{p, 0}$. This leads finally to the action (omitting the antifields, and taking $\alpha=0$ )

$$
\begin{aligned}
S_{0}= & P \dot{X}-\theta \not \partial \dot{\theta}-\frac{1}{2} P^{2}+b \dot{c}+4 b \dot{\theta} \theta_{1,0} \\
& +\sum_{q=1}^{\infty} \sum_{p=q}^{\infty} \bar{\lambda}^{p, q} \dot{\theta}_{p, q}+\lambda_{p, q} \dot{\bar{\theta}}^{p, q} .
\end{aligned}
$$

However, this action is not yet gauge fixed. We find a remaining gauge transformation of the form

$$
\begin{aligned}
\delta \theta & =\not b b \kappa_{1} \\
\delta X^{\mu} & =\theta \gamma^{\mu} \not b b \kappa_{1} \\
\delta \theta_{1,0} & =\frac{1}{2} P^{2} \kappa_{1} \\
\delta c & =-4 b \theta_{1} \not P \kappa_{1}
\end{aligned}
$$


where $\kappa_{1}$ is a commuting spinor parameter. So we still do not find a gauge fixed action.

We conclude that although the BS and DISP actions are describing the same physical variables, the former has not yet found a correct covariant gauge fixing procedure, while this is easy for the latter one.

\section{Acknowledgments}

This work was performed during a long period. Parts have been performed at the Theory Division of CERN. R.K. and A.V.P. are grateful for the hospitality there. Other parts were performed in the Physics Department of Leuven University, and E.B. would like to thank the members of this Institute for their hospitality.

We profited from many fruitful discussions, especially with J. Figueroa, J. Fisch, W. Siegel, W. Troost and J.W. van Holten. 


\section{A Notations and Conventions}

In this appendix we will explain the notation and conventions we are using in the paper. Often in this paper we delete vector indices. For instance the scalar product between two vectors $V_{\mu}, W_{\mu}$ is indicated as $V W$ instead of $V^{\mu} W_{\mu}$.

We also omit the spinor indices. Indicating explicit spinor indices we denote a positive-chiral spinor, like $\theta$, by $\theta^{\alpha}$, and a negative-chiral spinor, like $\theta_{1,0}$, by $\left(\theta_{1,0}\right)_{\alpha}$. However, we will never explicitly indicate these spinor indices. Note that a bar above a spinor, like in $\bar{\theta}^{0,0}$ does not refer to the Dirac conjugate but indicates that the corresponding spinor is an anti-ghost. The $\gamma$-matrices have their two indices both up or both down, and they are symmetric. One can therefore note the following identities

$$
\begin{aligned}
& \bar{\theta}^{p} \lambda_{p}=(-)^{p} \lambda_{p} \bar{\theta}^{p} \\
& \theta \gamma \theta_{2}=\gamma \theta \theta_{2}=\theta \theta_{2} \gamma .
\end{aligned}
$$

We use the following notations to indicate the system of ghosts, ghosts for ghosts, extra ghosts, antighosts and Lagrange multipliers corresponding to the kappa symmetries of the BS superparticle. The ghosts, ghosts for ghosts and extra ghosts are indicated by $\left\{\theta_{p, q}\right\}(p \geq q, q=0,1,, 2, \ldots)$; the antighosts are indicated by $\left\{\bar{\theta}^{p, q}\right\}(p \geq q, q=0,1,2 \ldots)$; the Lagrange multipliers corresponding to the non-minimal and extra ghosts are indicated by $\left\{\lambda_{p, q}\right\}(p \geq q, q=1,2 \ldots)$ and, finally, the Lagrange multipliers corresponding to the antighosts are indicated by $\left\{\bar{\lambda}^{p, q}\right\}(p \geq q, q=0,1,2, \ldots)$. All fields introduced above are spinor fields. Fields with lower indices have positive chirality for $p+q$ even, and negative chirality for $p+q$ odd, while the fields with upper indices have positive chirality for $p+q$ odd, and negative chirality for $p+q$ even.

We define the statistics of a Bose field to be + and that of a Fermi field to be - . If two fields $A, B$ have statistics $(-)^{a},(-)^{b}$, respectively, then the following rule applies: $A B=(-)^{a b} B A$. The statistics of the spinor fields we introduced above is given by:

$$
\begin{aligned}
& (-)^{p+q} \text { for } \bar{\theta}^{p, q}, \lambda_{p, q} \\
& (-)^{p+q+1} \text { for } \theta_{p, q}, \bar{\lambda}^{p, q} \text {. }
\end{aligned}
$$

In the DISP action the fields have another origin. In table 1 we give the fields with the general name in the first column, then the more specific name and the name as in the BS action. Finally there are the statistics, the ghost number and the ghost number of the antifield. Note that $\theta=\theta_{0}=\theta_{0,0}$ and $c^{a_{0}}=c^{a}, b_{a_{0}}=b_{a}$.

In the Batalin-Vilkovisky formulation one introduces for each field an antifield with opposite statistics and ghost number equal to

$$
g h\left(\Phi^{*}\right)=-g h(\Phi)-1
$$


Table 1: Fields in the DISP action

\begin{tabular}{|lllccc|}
\hline & DISP & BS & stat & $g h$ & $g h^{*}$ \\
\hline$\phi^{i}$ & $X^{\mu}$ & $X^{\mu}$ & + & 0 & -1 \\
& $P_{\mu}$ & $P_{\mu}$ & + & 0 & -1 \\
& $\theta_{p}$ & $\theta_{p, p}$ & - & 0 & -1 \\
& $\lambda^{p}$ & $\bar{\lambda}^{p, p}$ & - & 0 & -1 \\
\hline$\psi^{a}$ & $g$ & $g$ & + & 0 & -1 \\
& $\zeta^{p}$ & & - & 0 & -1 \\
& $\eta_{p}$ & & - & 0 & -1 \\
\hline$c^{a}$ & $c$ & $c$ & - & 1 & -2 \\
& $c^{p}$ & $\theta_{p+1, p}$ & + & 1 & -2 \\
& $c^{p^{\prime}}$ & $\lambda_{p+1, p+1}$ & + & 1 & -2 \\
\hline$c^{a_{p}}$ & $c^{q_{p}}$ & $\theta_{q+p+1, q}(--)^{p}$ & $(-)^{p}$ & $p+1$ & $-p-2$ \\
& $c^{q_{p}^{\prime}}$ & $\lambda_{q+p+1, q+1}(-)^{\frac{p(p-1)}{2}}$ & $(-)^{p}$ & $p+1$ & $-p-2$ \\
\hline$\check{c}^{a_{p+1}}$ & $\bar{c}^{q_{p+1}}$ & & $(-)^{p}$ & $p+1$ & $-p-2$ \\
& $\check{c}^{q_{p+1}^{\prime}}$ & & $(-)^{p}$ & $p+1$ & $-p-2$ \\
\hline$b_{a}$ & $b$ & $b$ & - & -1 & 0 \\
& $b_{p}$ & $\bar{\lambda}^{p+1, p}$ & + & -1 & 0 \\
& $b_{p^{\prime}}$ & $-\bar{\theta}^{p+1, p+1}$ & + & -1 & 0 \\
\hline$b_{a_{p}}$ & $b_{q_{p}}$ & $\lambda_{q+p+1, q}(-)^{p}$ & $(-)^{p}$ & $-(p+1)$ & $p$ \\
& $b_{q_{p}^{\prime}}$ & $-\bar{\theta}_{q+p+1, q+1}(-)^{\frac{p(p+1)}{2}}$ & $(-)^{p}$ & $-(p+1)$ & $p$ \\
\hline
\end{tabular}

An antibracket is defined between two functions $F$ and $G$ of the fields and antifields by

$$
(F, G)=\frac{\stackrel{\leftarrow}{\partial} F}{\partial \Phi^{A}} \frac{\vec{\partial} G}{\partial \Phi_{A}^{*}}-\frac{\stackrel{\leftarrow}{\partial} F}{\partial \Phi_{A}^{*}} \frac{\vec{\partial} G}{\partial \Phi^{A}}
$$

An extended action is defined (often called generating function, but this causes confusion with the generating function in the framework of canonical transformations, see appendix B). It first of all contains the action, i.e. the terms without antifields. Then it defines the BRST transformations using

$$
\delta_{B R S T}(\Lambda) f(\Phi)=\left.(f(\Phi), S)\right|_{\Phi^{*}=0} \Lambda=-\left.\Lambda(S, f(\Phi))\right|_{\Phi^{*}=0} .
$$

The invariance of the action under this transformation and the nilpotency of these transformations are equivalent to the 'master equation' $(S, S)=0$. This needs also the terms quadratic in antifields which correspond to the non-closure functions of the off-shell algebra.

We can also use the operator language. We define then the momentum $\Pi$ corresponding to the field $\Phi$ and its canonical bracket relations by

$$
\Pi=\frac{\overleftarrow{\partial} S_{0}}{\partial \dot{\Phi}} ; \quad[\Phi, \Pi]=1
$$


For first-order theories (at most linear in time derivatives) we can also express this as follows. We consider an extended action of the form

$$
S=\frac{1}{2} \Phi^{A} C_{A B} \dot{\Phi}^{B}-V(\Phi)+\Phi_{A}^{*} R(\Phi)+\mathcal{O}\left(\left(\Phi^{*}\right)^{2}\right)
$$

where $V(\Phi)$ does not contain time derivatives and $C_{A B}$ are constants. We can choose a graded antisymmetric $C$ (i.e. $C_{A B}=(-)^{A B+1} C_{B A}$ ) by adding a total derivative to the action. On the subset of fields where $C_{A B}$ is invertible $\left(C_{A B} C^{B C}=\delta_{A}^{C}\right)$ we define Dirac brackets by

$$
[F, G]=\frac{\overleftarrow{\partial} F}{\partial \Phi^{A}} C^{A B} \frac{\vec{\partial} G}{\partial \Phi^{B}}=[G, F](-)^{F G+1}
$$

Note that we use a unified notation for these brackets which can become commutators or anticommutators.

If also $R(\Phi)$ does not contain time derivatives, then the invariance of the action (or the master equation at $\Phi^{*}=0$ ) gives

$$
\int d t \dot{\Phi}^{A} C_{A B} R^{B}(\Phi)=0 \Rightarrow C_{A B} R^{B}(\Phi)=\frac{\vec{\partial} \Omega(\Phi)}{\partial \Phi^{A}}
$$

for some $\Omega(\Phi)$. This function is then the BRST operator in the sense that on the set of fields where $C_{A B}$ is invertible

$$
\delta_{B R S T}(\Lambda) \Phi=[\Phi, \Omega] \Lambda=\Lambda[\Omega, \Phi] .
$$

One can also obtain the BRST operator in other situations as is illustrated in appendix $\mathrm{C}$.

\section{B Canonical transformations}

In this appendix we repeat some essential formulae of canonical transformations from [31]. Full proofs and more properties are given in [32]. We also derive three lemmas involving these canonical transformations and which are frequently used in the paper.

Canonical transformations in the space of fields and antifields $\left(\Phi^{A}, \Phi_{A}^{*}\right)$ are defined as transformations from $\left(\Phi, \Phi^{*}\right)$ to $\left(\Phi^{\prime}, \Phi^{\prime *}\right)$ such that the antibrackets are left invariant. Therefore they also conserve the master equation $(S, S)=0$. They can always be obtained from a fermionic generating function $F\left(\Phi, \Phi^{\prime *}\right)$ for which the matrix

$$
\frac{\vec{\partial} \partial F\left(\Phi, \Phi^{\prime *}\right)}{\partial \Phi_{A}^{\prime *} \partial \Phi^{B}}
$$

is non-singular. (This is the " $F_{2}$ " in the terminology of canonical transformations.) The new variables are defined by

$$
\Phi^{\prime A}=\frac{\partial F\left(\Phi, \Phi^{\prime *}\right)}{\partial \Phi_{A}^{\prime *}} \quad \Phi_{A}^{*}=\frac{\partial F\left(\Phi, \Phi^{\prime *}\right)}{\partial \Phi^{A}} .
$$


We will use the following notation

$$
F\left(\Phi^{\prime *}, \Phi^{A}\right)=\Phi_{A}^{\prime *} \Phi^{A}+f\left(\Phi^{\prime *}, \Phi^{A}\right)
$$

where the first term includes a sum over all the fields. The gauge fixing can also be viewed as a canonical transformation with generating fermion

$$
f\left(\Phi, \Phi^{\prime *}\right)=\Psi(\Phi)
$$

which satisfies the non-degeneracy condition, because (B.1) is then the unit matrix.

We will now derive three lemmas involving these canonical transformations. The first two show how to eliminate auxiliary fields in this formalism. It is well known how to do this in the action and transformation laws. The power of these theorems lies in the fact that they also apply to non-closure functions and other expressions of higher order in antifields. The first lemma treats the case of auxiliary fields occurring linearly in the action, while the second deals with fields occurring quadratically.

Lemma B.1 We split the set of fields $\{\Phi\}$ in the "auxiliary ones" $\psi^{p}$ and $\pi_{p}$ and the remaining ones $\{\phi\}$. We consider then extended actions of the form

$$
S=\psi^{p} \pi_{p}+\psi^{p} A_{1 p}\left(\phi, \phi^{*}\right)+A_{2}^{p}\left(\phi, \phi^{*}\right) \pi_{p}+S_{0}
$$

where

$$
\frac{\partial S_{0}}{\partial \pi}=0 ;\left.\quad \frac{\partial S_{0}}{\partial \psi}\right|_{\psi^{*}=0}=0 .
$$

After canonical transformations we can put all these auxiliary fields and their antifields equal to zero and the resulting extended action is given by

$$
S=\left.S_{0}\right|_{\psi^{*}=\pi^{*}=0}-A_{2}^{p} A_{1 p} .
$$

In order to prove the lemma we first observe that from the master equation

$$
\frac{\partial}{\partial \pi_{p}}(S, S)=0
$$

one can derive the following identities

$$
\begin{aligned}
& \left(S, \psi^{p}\right)+\left(S, A_{2}^{p}\right)=0 \\
& \left(A_{2}^{p}, A_{2}^{q}\right)=0 .
\end{aligned}
$$

We now perform the following canonical transformation

$$
\begin{aligned}
\psi^{\prime p} & =\psi^{p}+A_{2}\left(\phi, \phi^{*}\right) \\
\psi_{p}^{\prime *} & =\psi_{p}^{*} \\
\phi^{\prime A} & =\exp \left(-\psi_{p}^{*} L_{A_{2}^{p}}\right) \phi^{A} \\
\phi_{A}^{\prime *} & =\exp \left(-\psi_{p}^{*} L_{A_{2}^{p}}\right) \phi_{A}^{*},
\end{aligned}
$$


where the Lie derivative is defined by

$$
L_{X} Y=(X, Y)
$$

To prove that this transformation is canonical we must show that the canonical brackets remain unchanged. To show that this is indeed the case, we obtain from eq.(B.10) that

$$
\begin{gathered}
\left(\psi^{p}, \exp \left(-\psi_{p}^{*} L_{A_{2}^{p}}\right) \phi^{A}\right)=-\exp \left(-\psi_{p}^{*} L_{A_{2}^{p}}\right)\left(A_{2}^{p}, \phi^{A}\right) \\
\left(T^{p}, \exp \left(-\psi_{p}^{*} L_{A_{2}^{p}}\right) \phi^{A}\right)=\exp \left(-\psi_{p}^{*} L_{A_{2}^{p}}\right)\left(A_{2}^{p}, \phi^{A}\right)
\end{gathered}
$$

which implies that $\psi^{\prime p}$ has zero brackets with $\phi^{\prime A}$ (and similarly for $\phi_{A}^{\prime *}$ ). That $\phi^{\prime A}$ and $\phi_{B}^{\prime *}$ then have canonical brackets follows from the previous result and by applying Jacobi identities. For instance,

$$
\frac{\partial}{\partial \psi_{p}^{*}}\left(\phi^{\prime A}, \phi_{B}^{\prime *}\right)=\left(\psi^{\prime p},\left(\phi^{\prime A}, \phi_{B}^{\prime *}\right)\right)=0
$$

and thus

$$
\left(\phi^{\prime A}, \phi_{B}^{\prime *}\right)=\left.\left(\phi^{\prime A}, \phi_{B}^{\prime *}\right)\right|_{\psi^{*}=0}=\left(\phi^{A}, \phi_{B}^{*}\right) .
$$

We have thus shown that the transformations defined above are canonical. Using the fact that brackets are invariant after canonical transformations we obtain from eq.(B.9) that in the new variables $\partial S / \partial \psi^{\prime *}=0$. Therefore the action after this canonical transformation (using the conditions eq.(B.6)) is of the form

$$
S=\psi^{\prime p} \pi_{p}+\psi^{\prime p} A_{1 p}-A_{2}^{p} A_{1 p}+\left.S_{0}\right|_{\psi^{*}=0}
$$

To bring the extended action into the final form given by the lemma we now perform a similar canonical transformation such that

$$
\pi_{p}^{\prime}=\pi_{p}+A_{1 p}
$$

To prove that this is a canonical transformation too, one should first prove that $\left(A_{1 p}, A_{1 q}\right)=0$ which indeed follows from the master equation. The same steps which were performed after the first canonical transformation now lead to an action where the auxiliary fields and their antifields occur only as $\psi^{\prime p} \pi_{p}^{\prime}$. This term can be integrated out of the path integral, which proves the lemma.

Lemma B.2 We split the set of fields $\{\Phi\}$ in the "auxiliary" boson $\lambda$ and the remaining ones $\{\phi\}$. We consider then extended actions of the form

$$
S=\lambda^{2}+\lambda A\left(\phi, \phi^{*}\right)+S_{0}
$$

where

$$
\frac{\partial S_{0}}{\partial \lambda}=0
$$


After canonical transformations we can put this auxiliary field and its antifield equal to zero and the resulting extended action is given by

$$
S=\left.S_{0}\right|_{\lambda^{*}=0}-\frac{1}{4} A^{2} .
$$

The proof proceeds as above.

We finally prove a third lemma. This lemma applies to the situation where a field is gauged away by an algebraic gauge condition.

Lemma B.3 Consider a theory containing fields $\lambda^{p}$ which occur at most linear in the extended action. The extended action can contain their antifields in an arbitrary way. If we fix some gauge symmetries by putting these fields equal to zero, then the resulting extended action is obtained by replacing in the minimal terms $\lambda^{p}$ by $-b^{* p}$ and $\lambda_{p}^{*}$ by $b_{p}$ where $b_{p}$ are antighosts for this symmetry.

The conditions of the lemma imply that the extended action can be written as

$$
S=U\left(\lambda^{*}, \phi, \phi^{*}\right)-\lambda^{p} T_{p}\left(\lambda^{*}, \phi, \phi^{*}\right)+b^{* p} d_{p}
$$

where $\phi$ refers again to all unspecified fields and the last term is the nonminimal term containing the antifields of the antighosts and the Lagrange multipliers. Then the gauge fixing which puts the fields equal to zero is defined by the gauge fermion $\Psi=\lambda^{p} b_{p}$. This results in

$$
S=U\left(\lambda^{*} \rightarrow \lambda^{*}+b\right)-\lambda^{p} T_{p}\left(\lambda^{*} \rightarrow \lambda^{*}+b\right)+b^{* p} d_{p}+\lambda^{p} d_{p} .
$$

It will be useful to split the $T_{p}$ term in

$$
T_{p}\left(\lambda^{*} \rightarrow \lambda^{*}+b\right)=T_{p}\left(\lambda^{*} \rightarrow b\right)+T_{p}{ }^{q} \lambda_{q}^{*}
$$

for some new function $T_{p}^{q}\left(\lambda^{*}, b, \phi, \phi^{*}\right)$. Now we can apply the first lemma, identifying $\psi$ with $\lambda$, $\pi$ with $d$ and

$$
\begin{aligned}
& A_{1 p}=-T_{p}\left(\lambda^{*} \rightarrow b\right) ; \quad A_{2}^{p}=b^{* p} \\
& S_{0}=U\left(\lambda^{*} \rightarrow \lambda^{*}+b\right)-\lambda^{p} T_{p}^{q} \lambda_{q}^{*} .
\end{aligned}
$$

One can check that $S_{0}$ satisfies the conditions eq.(B.6). The result is thus

$$
S=U\left(\lambda^{*} \rightarrow b\right)+b_{p}^{*} T_{p}\left(\lambda^{*} \rightarrow b\right),
$$

which is eq.(B.21) with $\lambda^{p} \rightarrow-b^{* p}$ and $\lambda_{p}^{*} \rightarrow b_{p}$.

The result of this lemma, making the connection between the Lagrange multipliers $\lambda^{p}$ and antighosts $b_{p}$ is also used in the relation between Hamiltonian and Lagrangian BRST [27] and for providing regulators in the calculation of anomalies [30]. 


\section{Example : the antisymmetric tensor.}

In this appendix we would like to illustrate some of the techniques we have been using in the paper by means of the antisymmetric tensor. In this example we will first exhibit the solution to the master equation and show the physical states. We will furthermore illustrate the two-step gauge fixing procedure which was used in the quantisation of the BS superparticle in subsection 2. In this appendix we will use as four-dimensional metric $(-+++)$.

The classical action for the antisymmetric tensor is given by

$$
S_{0}=-\frac{1}{6} B_{\mu \nu \rho} B^{\mu \nu \rho}
$$

where

$$
B_{\mu \nu \rho}=\partial_{\mu} B_{\nu \rho}+\partial_{\rho} B_{\mu \nu}+\partial_{\nu} B_{\rho \mu}
$$

This action is invariant under the gauge transformations $\delta B_{\mu \nu}=\partial_{\mu} \Lambda_{\nu}-\partial_{\nu} \Lambda_{\mu}$. The physical states are by definition solutions of the field equations modulo gauge transformations. The examination is most easily performed by going to momentum space.

We first prove that $k^{2}=0$. If $k^{2} \neq 0$ then we can choose either a frame where $k$ is in the 0 direction (if $k^{2}<0$ ) or in the 1 direction (if $k^{2}>0$ ). In each case the gauge transformation of $k^{\mu} B_{\mu \nu}$ for $\nu$ in the other three directions is of the form $\delta\left(k^{\mu} B_{\mu \nu}\right)=k^{2} \Lambda_{\nu}$. So we can choose a gauge where these objects are equal to zero. Then the remaining part of the field equations is $k^{2} B_{\mu \nu}=0$ and there is no solution.

As $k^{2}=0$ we can choose a light-cone basis with $V^{\mu} W_{\mu}=V^{+} W^{-}+$ $V^{-} W^{+}+V^{i} V^{i}$ and $k^{\mu}=\delta_{+}^{\mu} k$. The field equations are reduced to $k_{[\mu} B_{\nu]+}=0$ eliminating $B_{i+}$. The non-zero gauge transformations are $\delta B_{-+}=k \Lambda_{+}$and $\delta B_{-i}=k \Lambda_{i}$. These components are thus also eliminated and the only physical degree of freedom is the antisymmetric $B_{i j}$. We are thus left with one massless field.

Following the $\mathrm{BV}$ procedure we define the extended action

$$
S=-\frac{1}{6} B_{\mu \nu \rho} B^{\mu \nu \rho}+B_{\mu \nu}^{*} \partial^{\mu} c^{\nu}+c_{\mu}^{*} \partial^{\mu} c
$$

whose antibracket cohomology represents the physical state.

\section{C.1 The usual gauge fixing}

The usual way to perform the gauge fixing is introducing all the fields in table 2 . We thus add to the extended action the non-minimal terms

$$
S_{n m}=b^{* \mu} \lambda_{\mu}+b^{*} \lambda+b^{*} \lambda^{\prime}
$$

and choose the gauge fermion to be

$$
\Psi=-2 b_{\mu} \partial_{\nu} B^{\mu \nu}+b_{\mu} \lambda^{\mu}+b \partial_{\mu} c^{\mu}+b_{\mu} \partial^{\mu} b^{\prime}-\lambda^{\prime} b .
$$


Table 2: Fields in the BV quantisation of the antisymmetric tensor

\begin{tabular}{ccccc}
\hline & & $\bar{B}_{\mu \nu}$ & & \\
& $b_{\mu}$ & & $c^{\mu}$ & \\
$b$ & & $b^{\prime}$ & & $c$ \\
& & & & \\
& & & & \\
& $\lambda^{\mu}$ & & & \\
$\lambda$ & & $\lambda^{\prime}$ & &
\end{tabular}

After diagonalisation of the $\lambda_{\mu}, \lambda$ and $\lambda^{\prime}$ terms by applying Lemma B.19 the extended action becomes

$$
\begin{aligned}
S= & -\frac{1}{2} \partial_{\mu} B_{\nu \rho} \partial^{\mu} B^{\nu \rho}-\partial_{\mu} b_{\nu} \partial^{\mu} c^{\nu}-\partial_{\mu} b \partial^{\mu} c-\frac{1}{4} \partial_{\mu} b^{\prime} \partial^{\mu} b^{\prime} \\
& +B_{\mu \nu}^{*} \partial^{\mu} c^{\nu}+c_{\mu}^{*} \partial^{\mu} c+b^{*} \partial_{\mu} b^{\mu}+b^{\prime *} \partial_{\mu} c^{\mu}+b_{\mu}^{*}\left(\partial_{\nu} B^{\mu \nu}-\frac{1}{2} \partial^{\mu} b^{\prime}\right) \\
& -\frac{1}{4} b^{* \mu} b_{\mu}^{*}+b^{\prime *} b^{*} \\
& +\lambda^{\mu} \lambda_{\mu}+\lambda \lambda^{\prime}
\end{aligned}
$$

The last line implies that the $\lambda^{\mu}, \lambda$ and $\lambda^{\prime}$ can be omitted as these are trivial systems. The first line shows that this action is gauge-fixed and the field equations imply that all fields are massless. We then use the same decomposition in $(+,-, i)$ components as above and the BRST transformations, determined by the second line are given by

$$
\begin{aligned}
& \delta B^{+-}=\frac{1}{2} k c^{-} ; \quad \delta B^{+i}=\frac{1}{2} k c^{i} \\
& \delta c^{+}=k c ; \quad \delta b=k b^{-} ; \quad \delta b^{\prime}=k c^{-} \\
& \delta b^{i}=k B^{i-} ; \delta b^{+}=k\left(B^{+-}-\frac{1}{2} b^{\prime}\right)
\end{aligned}
$$

The remaining component is again $B_{i j}$.

\section{C.2 Two-step procedure, gauge 1}

We now illustrate the two-step procedure. To start, we try a gauge fixing using as non-minimal terms only

$$
S_{n m}=b^{* \mu} \lambda_{\mu}+b^{*} \lambda
$$

and choosing

$$
\Psi=b_{\mu} \partial_{\nu} B^{\mu \nu}+b \partial_{\mu} c^{\mu}
$$


We thus get the extended action

$$
\begin{aligned}
S= & -\frac{1}{6} B_{\mu \nu \rho} B^{\mu \nu \rho}+b_{[\mu} \partial_{\nu]} \partial^{\mu} c^{\nu}+b \partial_{\mu} \partial^{\mu} c+\lambda_{\mu} \partial_{\nu} B^{\mu \nu}+\lambda \partial_{\mu} c^{\mu} \\
& +B_{\mu \nu}^{*} \partial^{\mu} c^{\nu}+c_{\mu}^{*} \partial^{\mu} c+b^{* \mu} \lambda_{\mu}+b^{*} \lambda
\end{aligned}
$$

We next observe that this action still has the following two symmetries

$$
\delta b_{\mu}=\partial_{\mu} \eta ; \quad \delta \lambda_{\mu}=\partial_{\mu} \xi
$$

We try a two-step gauge fixing procedure. We introduce for both symmetries a ghost, antighost and Lagrange multiplier, which we call

$$
g, g^{\prime}, \lambda^{\prime} ; \quad \gamma, \gamma^{\prime}, b^{\prime}
$$

The first two fields are bosonic, the next three fermionic, and the last field is again bosonic. We have thus added the following terms to the extended action :

$$
b_{\mu}^{*} \partial^{\mu} g+\lambda_{\mu}^{*} \partial^{\mu} \gamma-g^{*} \gamma+g^{\prime *} \lambda^{\prime}+\gamma^{\prime *} b^{\prime} .
$$

The first two terms introduce the extra symmetry transformations as new ghost terms. The third term is necessary to get $(S, S)=0$ for the total extended action. The last two are usual non-minimal terms. We could then fix these two symmetries by introducing the gauge fermion

$$
\Psi^{\prime}=b_{\mu} \partial^{\mu} g^{\prime}+\left(\partial^{\mu} \gamma^{\prime}\right) \lambda_{\mu}
$$

We perform still a canonical transformation

$$
\tilde{\lambda}^{\mu}=\lambda^{\mu}+\partial^{\mu} g ; \quad g^{*}=\tilde{g}^{*}-\partial^{\mu} \lambda_{\mu}^{*} .
$$

The extended action then becomes

$$
\begin{aligned}
S= & -\frac{1}{6} B_{\mu \nu \rho} B^{\mu \nu \rho}+b_{[\mu} \partial_{\nu]} \partial^{\mu} c^{\nu}+b \partial_{\mu} \partial^{\mu} c+\lambda_{\mu} \partial_{\nu} B^{\mu \nu}+\lambda \partial_{\mu} c^{\mu} \\
& +\partial_{\mu} g^{\prime} \lambda^{\mu}+b_{\mu} \partial^{\mu} \lambda^{\prime}+\partial^{\mu} b^{\prime} \lambda_{\mu}-\partial_{\mu} g \partial^{\mu} b^{\prime}+\partial^{\mu} \gamma^{\prime} \partial_{\mu} \gamma \\
& +B_{\mu \nu}^{*} \partial^{\mu} c^{\nu}+c_{\mu}^{*} \partial^{\mu} c+b^{* \mu} \lambda_{\mu}+b^{*} \lambda \\
& -g^{*} \gamma+g^{\prime *} \lambda^{\prime}+\gamma^{\prime *} b^{\prime} .
\end{aligned}
$$

This action is now gauge-fixed, and the reader can easily verify that the BRST cohomology together with the field equations eliminates all fields except one massless scalar in $B_{i j}$.

We got thus the correct result in this two-step gauge fixing procedure. The only point where we did not just perform canonical transformations was when we added the terms eq.(C.13). Using at that point already the variables eq.(C.15) the first term is absorbed in the $b^{* \mu} \lambda_{\mu}$ term of eq.(C.10), while the second term gets absorbed in the third term. The remaining three terms are of the standard trivial type showing that all new variables do not contribute to the antibracket cohomology. It was thus clear that this second step gauge fixing did not change our physical states. It just amounted to addition of trivial systems, an allowed procedure. 


\section{C.3 Two-step procedure, gauge 2}

We now consider another gauge, more similar to the one which we considered before. So we take again eq.(C.5) but without the $b^{\prime}$ and $\lambda^{\prime}$ terms, thus :

$$
\Psi=-2 b_{\mu} \partial_{\nu} B^{\mu \nu}+b_{\mu} \lambda^{\mu}+b \partial_{\mu} c^{\mu} .
$$

We can still eliminate $\lambda_{\mu}$, and obtain as extended action

$$
\begin{aligned}
S= & -\frac{1}{2} \partial_{\mu} B_{\nu \rho} \partial^{\mu} B^{\nu \rho}-2 \partial_{[\mu} b_{\nu]} \partial^{\mu} c^{\nu}-\partial_{\mu} b \partial^{\mu} c+\partial_{\mu} c^{\mu} \lambda \\
& +B_{\mu \nu}^{*} \partial^{\mu} c^{\nu}+c_{\mu}^{*} \partial^{\mu} c+b^{\star} \lambda+b_{\mu}^{*} \partial_{\nu} B^{\mu \nu}-\frac{1}{4} b^{* \mu} b_{\mu}^{*}
\end{aligned}
$$

Now there is only one extra gauge transformation of this 'gauge-fixed' action : $\delta b_{\mu}=\partial_{\mu} \eta$. We thus add

$$
S_{\text {extra }}=b^{* \mu} \partial_{\mu} g+b^{\prime *} \lambda^{\prime}
$$

(the new antighost is now already called $b^{\prime}$ ). After the choice of the gauge fermion

$$
\Psi^{\prime}=b^{\mu} \partial_{\mu} b^{\prime}-\lambda^{\prime} b
$$

we can use again lemma B.1 for eliminating $\lambda$ and $\lambda^{\prime}$, and after the redefinition $\left(\tilde{b}^{\prime}=b^{\prime}-2 g\right)$ we get again eq.(C.6) with an extra term

$$
+\partial_{\mu} g \partial^{\mu} g
$$

As $g$ does not appear in the BRST transformations, it is also a (ghost number zero) solution of the BRST cohomology!

The step which was not a canonical transformation was adding the terms eq.(C.19). This time these were not just trivial terms, and introduced the new 'physical' variable $g$. This is completely analogous to the creation of the extra solutions of the cohomology which we found in section 2 after fixing the Stueckelberg symmetries. From subsection C.1 we know what should have been a correct procedure after eq.(C.18). We should have added a non-minimal system $b^{\prime *} \lambda^{\prime}$ to the extended action and perform a canonical transformation using the gauge fermion $\Psi=b_{\mu} \partial^{\mu} b^{\prime}-\lambda^{\prime} b$. This would lead to the same correct result as in subsection C.1.

We conclude that one can do the gauge fixing in two steps if one adds only trivial systems in starting the second step (see subsection C.2). New solutions of the cohomology can be created if this requirement is not satisfied as in the last subsection.

\section{References}

[1] M.B. Green and J.H. Schwarz, Phys. Lett. B136 (1984) 367. 
[2] L.D. Faddeev and V.N. Popov, Phys. Lett. 25B (1967) 29.

[3] I.A. Batalin and G.A. Vilkovisky, Phys. Rev. D28 (1983) 2567 (E:D30 (1984) 508).

[4] L. Brink and J.H. Schwarz, Phys. Lett. B100 (1981) 310.

[5] W. Siegel, Phys. Lett. 128B (1983) 397.

[6] W. Siegel, in 'Strings 89', eds. R. Arnowitt et al., World Scientific, 1990.

[7] A. Miković, M. Roček, W. Siegel, P. van Nieuwenhuizen, J. Yamron and A.E. van de Ven, Phys. Lett. B235 (1990) 106.

[8] M.B. Green and C.M. Hull, Nucl. Phys. B344 (1990) 115.

[9] M.B. Green and C.M. Hull, Mod. Phys. Lett. A5 (1990) 1399 and contribution to the conference "Strings '90".

[10] E.A. Bergshoeff, R. Kallosh and A. Van Proeyen, Phys. Lett. B251 (1990) 128

[11] R. Kallosh, Phys. Lett. B251 (1990) 134.

[12] F. Eßler, E. Laenen, W. Siegel and J.P. Yamron, preprint ITP-SB-90-76; F. Eßler, M. Hatsuda, E. Laenen, W. Siegel, J.P. Yamron, T. Kimura and A. Miković, preprint ITP-SB-90-77.

[13] J. Fisch, M. Henneaux, J. Stasheff and C. Teitelboim, Commun. Math. Phys. 120 (1989) 379;

J. Fisch and M. Henneaux, Commun. Math. Phys. 128 (1990) 627.

[14] J.M.L. Fisch, 'On the Batalin-Vilkovisky antibracket-antifield BRST formalism and its applications', Ph.D thesis, ULB TH2/90-01.

[15] U. Lindström, M. Roček, W. Siegel, P. van Nieuwenhuizen and A.E. van de Ven, Phys. Lett. B224 (1989) 285; B228 (1989) 53

M. Roček, W. Siegel, P. van Nieuwenhuizen and A.E. van de Ven, Phys. Lett. B227 (1989) 87.

[16] F. Bastianelli, G.W. Delius and E. Laenen, Phys. Lett. B229 (1989) 223.

[17] E. Bergshoeff and R. Kallosh, Phys. Lett. B240 (1990) 105.

[18] M.B. Green and C.M. Hull, in 'Strings 89', eds. R. Arnowitt et al., World Scientific, 1990.

[19] J.M.L. Fisch and M. Henneaux, preprint ULB-TH2/89-04.

[20] M.B. Green and C.M. Hull, Phys. Lett. B229 (1989) 215. 
[21] I. Bars and R. Kallosh, Phys. Lett. B233 (1989) 117.

[22] L. Brink, Phys. Lett. B241 (1990) 19.

[23] R. Kallosh, Phys. Lett. B195 (1987) 369.

[24] J.W. van Holten and J. Kowalski-Glikman, Nucl. Phys. proc. supplement B6 (1989) 192.

[25] T. Kugo and K. Ojima, Suppl.prog. Theor. Phys. 66 (1979) 1.

[26] P. van Nieuwenhuizen, Phys. Rev. D24 (1981) 3315.

[27] W. Siegel, IJMPA 4 (1989) 3951;

J.M.L. Fisch and M. Henneaux, Phys. Lett. B226 (1989) 80;

C. Battle, J. Gomis, J. París and J. Roca, Phys. Lett. B224 (1989) 288; Nucl. Phys. B329 (1990) 139.

[28] I.A. Batalin and G.A. Vilkovisky, J. Math. Phys. 26 (1985) 172.

[29] I.A. Batalin, R.E. Kallosh and A. Van Proeyen, in 'Quantum Gravity', eds. M.A. Markov, V.A. Berezin and V.P. Frolov, World Scientific, 1987.

[30] A. Diaz, W. Troost, P. van Nieuwenhuizen and A. Van Proeyen, Int. J. Mod Phys. A4 (1989) 3959.

[31] I.A. Batalin and G.A. Vilkovisky, Nucl. Phys. B234 (1984) 106.

[32] W. Troost, P. van Nieuwenhuizen and A. Van Proeyen, Nucl. Phys. B333 (1990) 727. 